\title{
MAXIMAL SUBBUNDLES OF PARABOLIC VECTOR BUNDLES*
}

\author{
USHA N. BHOSLE ${ }^{\dagger}$ AND INDRANIL BISWAS ${ }^{\dagger}$
}

\begin{abstract}
Let $X$ be a complex irreducible smooth projective curve of genus at least two and $M(r, d)$ a moduli space of stable parabolic vector bundles over $X$ of rank $r$ and degree $d$ with a fixed parabolic structure. For any parabolic bundle $E_{*} \in M(r, d)$ and a subbundle $F \subset E$ of rank $r^{\prime}$ and fixed induced parabolic structure, set $s^{\text {par }}\left(E_{*}, F_{*}\right):=d r^{\prime}-\operatorname{deg}(F) r$, where $F_{*}$ is $F$ equipped with the induced parabolic structure. If $E_{*}$ has a subbundle of rank $r^{\prime}$ with the fixed induced parabolic structure, then let $s_{r^{\prime}}^{\text {par }}\left(E_{*}\right)$ be the minimum of $s^{\text {par }}\left(E_{*}, F_{*}\right)$ taken over all such subbundles $F$. We investigate the strata of $M(r, d)$ defined by values of $s_{r^{\prime}}^{p a r}\left(E_{*}\right)$.
\end{abstract}

Key words. Maximal subbundle, parabolic bundle, stability

AMS subject classifications. 14F05, 14H60

1. Introduction. Let $X$ be a connected smooth projective curve defined over $\mathbb{C}$ of genus $g$, with $g \geq 2$, and $P$ a finite set of distinct closed points of $X$. The canonical line bundle of $X$ will be denoted by $K$, and $K(P)$ will denote the line bundle $K \otimes \mathcal{O}_{X}(P)$.

Let $M(r, d)$ denote the moduli space of all stable parabolic vector bundles $E_{*}$ over $X$ of rank $r$ and degree $d$ with fixed parabolic structures over $P$. We will assume that all the parabolic weights are rational numbers. For parabolic vector bundles $E_{*}$ and $F_{*}$, let $\operatorname{Hom}\left(E_{*}, F_{*}\right)$ (respectively, $\operatorname{Hom}_{s t}\left(E_{*}, F_{*}\right)$ ) denote the sheaf of parabolic (respectively, strongly parabolic) homomorphisms from $E_{*}$ to $F_{*}$ (see Definition 2.2).

By a general parabolic vector bundle we will mean a parabolic bundle corresponding to a general point of $M(r, d)$ for some $r$ and $d$. We have the following analogue of Yoneda pairing.

Proposition 1.1 (Proposition 3.3). (1) Let $E_{*}$ and $F_{*}$ be general stable parabolic vector bundles. Then the cup product pairing

$$
H^{1}\left(\operatorname{Hom}\left(E_{*}, E_{*}\right)\right) \otimes H^{0}\left(\operatorname{Hom}\left(E_{*}, F_{*}\right)\right) \longrightarrow H^{1}\left(\operatorname{Hom}\left(E_{*}, F_{*}\right)\right)
$$

is identically zero.

(2) Let the assumptions be as in (1). The pairing of vector spaces (defined by composition of homomorphisms)

$H^{0}\left(H o m\left(E_{*}, F_{*}\right)\right) \otimes H^{0}\left(H_{o m}\left(F_{*}, E_{*} \otimes K(P)\right)\right) \longrightarrow H^{0}\left(H o m_{s t}\left(E_{*}, E_{*} \otimes K(P)\right)\right)$ is identically zero.

For a vector bundle $E$, let $\mu_{E}:=\operatorname{degree}(E) / \operatorname{rank}(E)$ be the slope of $E$.

Theorem 1.2 (Theorem 4.2). Let $A_{*}$ and $B_{*}$ be two general stable parabolic vector bundles over $X$. Then
(A) (1)
$H^{0}\left(\operatorname{Hom}\left(A_{*}, B_{*}\right)\right)=0$
if
(A) (2):
$H^{1}\left(\operatorname{Hom}\left(A_{*}, B_{*}\right)\right)=0$
if
$\mu_{B}-\mu_{A} \leq g-1+t_{A, B}$,
(B) (1): $H^{0}\left(\operatorname{Hom}_{s t}\left(A_{*}, B_{*}\right)\right)=0$ if $\mu_{B}-\mu_{A} \leq g-1+t_{A, B}+t_{A, B}^{0}$,
(B) (2): $H^{1}\left(\operatorname{Hom}_{s t}\left(A_{*}, B_{*}\right)\right)=0$ if $\mu_{B}-\mu_{A} \geq g-1+t_{A, B}+t_{A, B}^{0}$.

\footnotetext{
* Received January 14, 2004; accepted for publication October 26, 2005.

†School of Mathematics, Tata Institute of Fundamental Research, Homi Bhabha Road, Mumbai 400005, India (usha@math.tifr.res.in; indranil@math.tifr.res.in).
} 
Here $t_{A, B}$ and $t_{A, B}^{0}$ are rational numbers determined by the parabolic structures on $A_{*}$ and $B_{*}$ (see Eqn. (2.3) for the definition).

This result was proved earlier for ordinary vector bundles by Hirschowitz [Hi]. In case of ordinary vector bundles, the statements $(A)(1)$ and $(A)(2)$ are equivalent under Serre duality and one needs to prove only one statement. This is no longer true for parabolic vector bundles, hence both the statements have to be proved separately. The statements $(B)(1),(B)(2)$ follow from $(A)(2),(A)(1)$ respectively by duality. It may be noted that the known proofs of the theorem in the vector bundle case ([Hi], [RT]) do not generalize to the parabolic case, one of the problems being that the parabolic degrees are not integers.

Let $E_{*}$ be a parabolic vector bundle of rank $r$, degree $d$ and rational parabolic weights $\alpha_{i}(p), p \in P$, with multiplicities $n_{i}(p)$. Set $w t E=\sum_{p} \sum_{i} \alpha_{i}(p) n_{i}(p)$. We fix the parabolic data of the induced parabolic structure of subbundles of $E_{*}$ of fixed rank. So let $E_{*}^{\prime}$ denote a parabolic subbundle of $E_{*}$ of (fixed) rank $r^{\prime}$, (non-fixed) degree $d^{\prime}$, and weights $\alpha_{i}(p), p \in P$, with (fixed) multiplicities $n_{i}^{\prime}(p) \leq n_{i}(p)$, where $n_{i}^{\prime}(p)=0$ if $\alpha_{i}(p)$ is not a weight of $E_{*}^{\prime}$; the parabolic structure on $E_{*}^{\prime}$ is the one induced by $E_{*}$. Let $E_{*}^{\prime \prime}=E_{*} / E_{*}^{\prime}$ and $r^{\prime \prime}=r-r^{\prime}=\operatorname{rank}\left(E_{*}^{\prime \prime}\right)$. Define $s^{\text {par }}\left(E_{*}, E_{*}^{\prime}\right):=r^{\prime} d-r d^{\prime}$.

Definition 1.3. Fix integers $r^{\prime}$ and $\left\{n_{i}^{\prime}(p)\right\}_{p \in P}$ with $1 \leq r^{\prime}<r$ and $0 \leq$ $n_{i}^{\prime}(p) \leq n_{i}(p)$ with $\sum_{i} n_{i}^{\prime}(p)=r^{\prime}$ for all $p \in P$. Set $n_{i}^{\prime \prime}(p)=n_{i}(p)-n_{i}^{\prime}(p)$ and $t_{n^{\prime}, n^{\prime \prime}}=\sum_{p \in P} \sum_{i>j} n_{i}^{\prime}(p) n_{j}^{\prime \prime}(p)$ (in $t_{n^{\prime}, n^{\prime \prime}}$, the suffix $n^{\prime}$ stands for the tuple $\left(\left(n_{i}^{\prime}(p)\right)_{i, p}\right)$ and the suffix $n^{\prime \prime}$ stands for the tuple $\left.\left(\left(n_{i}^{\prime \prime}(p)\right)_{i, p}\right)\right)$. If a parabolic vector bundle $E_{*}$ has a parabolic subbundle $E_{*}^{\prime}$ of rank $r^{\prime}$ and weight $\alpha_{i}(p)$ of multiplicity $n_{i}^{\prime}(p), p \in P$, then we define

$$
s_{r^{\prime}}^{p a r}\left(E_{*}\right):=\min s^{p a r}\left(E_{*}, E_{*}^{\prime}\right),
$$

where the minimum is taken over all parabolic subbundles $E_{*}^{\prime} \subset E_{*}$ of rank $r^{\prime}$ and weight $\alpha_{i}(p)$ of multiplicity $n_{i}^{\prime}(p), p \in P$.

One has $s_{r^{\prime}}^{p a r}\left(E_{*}\right) \equiv r^{\prime} d \bmod r$. We prove that there is an upper bound

$$
s_{r^{\prime}}^{\text {par }}\left(E_{*}\right) \leq r^{\prime} r^{\prime \prime}\left(g+t_{n^{\prime}, n^{\prime \prime}}\right) .
$$

We use the correspondence between parabolic vector bundles on $X$ and vector bundles equipped with the action of the Galois group on a suitable normal covering of $X$ to get this upper bound.

The stability condition of $E_{*}$ gives a lower bound on $s_{r^{\prime}}^{p a r}\left(E_{*}\right), E_{*} \in M(r, d)$. We prove that for a general parabolic bundle $E_{*}$, either $E$ has no subbundle of given type or one has

$$
s_{r^{\prime}}^{p a r}\left(E_{*}\right) \geq r^{\prime} r^{\prime \prime}\left(g-1+t_{n^{\prime}, n^{\prime \prime}}\right) .
$$

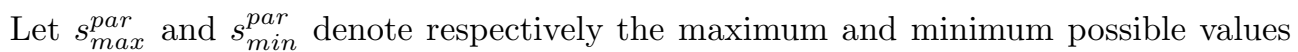
of $s_{r^{\prime}}^{p a r}\left(E_{*}\right)$, where the maximum (or minimum) is taken by moving $E_{*}$ over a moduli space of stable parabolic bundles (fixing $r^{\prime}$ and the induced parabolic structure of the subbundle).

Let $s$ denote an integer such that

$$
s_{\min }^{\text {par }} \leq s \leq s_{\max }^{\text {par }}, \quad s \equiv r^{\prime} d \bmod r .
$$

Let $M_{r^{\prime}, s}$ be the subset of $M(r, d)$ defined by

$$
M_{r^{\prime}, s}=\left\{E_{*} \in M(r, d) \mid s_{r^{\prime}}^{\text {par }}\left(E_{*}\right)=s\right\} .
$$


For a fixed $E_{*}$ and fixed $\alpha_{i}^{\prime}(p), n_{i}^{\prime}(p)$, let $A_{r^{\prime}, d^{\prime}}^{p a r}\left(E_{*}\right):=\left\{E_{*}^{\prime} \mid E_{*}^{\prime} \subset E_{*}\right.$ parabolic subbundle of rank $r^{\prime}$, degree $d^{\prime}$ and weights $\alpha_{i}^{\prime}(p)$ with multiplicities $\left.n_{i}^{\prime}(p), p \in P\right\}$.

THEOREM 1.4. (1) If $s \leq r^{\prime} r^{\prime \prime}\left(g-1+t_{n^{\prime}, n^{\prime \prime}}\right)$ and $M_{r^{\prime}, s}$ is nonempty, then

$$
\operatorname{dim} M_{r^{\prime}, s}=\operatorname{dim} M(r, d)+s-r^{\prime} r^{\prime \prime}\left((g-1)+t_{n^{\prime}, n^{\prime \prime}}\right) .
$$

A general element $E_{*}$ in $M_{r^{\prime}, s}$ has only finitely many subbundles of rank $r^{\prime}$, degree $d^{\prime}$ and with given induced parabolic structure, i.e., $\operatorname{dim} A_{r^{\prime}, d^{\prime}}^{\text {par }}\left(E_{*}\right)=0$.

(2) If $s \geq r^{\prime} r^{\prime \prime}\left((g-1)+t_{n^{\prime}, n^{\prime \prime}}\right)$, then

$$
\operatorname{dim} A_{r^{\prime}, d^{\prime}}^{\text {par }}\left(E_{*}\right) \leq s-r^{\prime} r^{\prime \prime}\left((g-1)+t_{n^{\prime}, n^{\prime \prime}}\right)
$$

and

(3) (A) Let $w=\sum_{p} \sum_{i} \alpha_{i}(p) n_{i}(p), w^{\prime}=\sum_{p} \sum_{i} \alpha_{i}(p) n_{i}^{\prime}(p), w^{\prime \prime}=w-w^{\prime}$. Assume that

$$
g \geq 1+\left(r^{\prime \prime} p_{0}-\left[w^{\prime \prime}\right]\right) / r^{\prime},
$$

where $p_{0}$ is the number of parabolic points and $\left[w^{\prime \prime}\right]$ denotes the integral part of $w^{\prime \prime}$. Then $M_{r^{\prime}, s}$ is nonempty if and only if $s_{0} \leq s \leq s_{\max }^{p}$ and $s \equiv r^{\prime} d \bmod r$, where $s_{0}$ is the unique integer satisfying

$$
r w^{\prime}-r^{\prime} w<s_{0} \leq r+r w^{\prime}-r^{\prime} w, s_{0} \equiv r^{\prime} d \bmod r .
$$

(B) If $r^{\prime}=r-1$, then the conclusion of (A) holds for all $g \geq 2$.

Note that from part 2 of Theorem 1.4 it follows that

$$
s_{\max }^{p a r}=r^{\prime} r^{\prime \prime}\left(g-1+t_{n^{\prime}, n^{\prime \prime}}\right)+\epsilon,
$$

where $\epsilon$ is the unique integer $0 \leq \epsilon \leq r-1$ with $s_{\max }^{p a r} \equiv r^{\prime} d \bmod r$.

The condition in Theorem 1.4.3(A) is used to prove that $M_{r^{\prime}, s_{0}}$ is nonempty. This is the most difficult part (Proposition 5.6). The nonemptiness of $M_{r^{\prime}, s}, s>s_{0}$ is deduced from the nonemptiness of $M_{r^{\prime}, s_{0}}$ using elementary transformations and dimension estimates (Lemmas 5.4, 5.2, 5.3, Proposition 5.5). Some of the subsets $M_{r^{\prime}, s}, s>s_{0}$ could possibly be nonempty under weaker (or different) conditions than those in 3(A) (for example, see Proposition 4.3).

The layout of the paper is as follows. In Section 2, all the general results on parabolic bundles needed in the paper are given. The upper bound on $s_{r^{\prime}}^{p a r}$ and the analogue of Yoneda pairing are proved in Section 3. Theorem 1.2 is proved in Section 4. The final Section 5 deals with maximal parabolic subbundles of parabolic vector bundles ending with the proof of Theorem 1.4.

2. Parabolic vector bundles. Let $X$ be a connected smooth projective curve of genus $g$, with $g \geq 2$ over the field of complex numbers and $P$ a finite set of distinct closed points of $X$. Let $p_{0}$ be the number of points $p \in P$.

For a vector bundle $E$ on $X$, we denote the rank, degree and slope of $E$ by $r(E)$, $d(E)$ and $\mu_{E}$ respectively.

Definition 2.1. Let $P$ be a finite set of distinct closed points of $X$. A parabolic structure on a vector bundle $E$ on $X$ at $p \in P$ consists of the following. 
(1) A flag of the fiber of $E$ at $p$

$$
E_{p}=E_{p, 1} \supset E_{p, 2} \supset \cdots \supset E_{p, l_{p}} \supset E_{p, l_{p}+1}=0 .
$$

(2) A sequence of real numbers

$$
0 \leq \alpha_{1}(p)<\alpha_{2}(p)<\cdots<\alpha_{l_{p}}(p)<1 .
$$

These numbers $\left\{\alpha_{i}(p)\right\}$ are called parabolic weights at $p$. The integer

$$
n_{i}(p)=\operatorname{dim} E_{p, i} / E_{p, i+1},
$$

$1 \leq i \leq l_{p}$ is called the multiplicity of $\alpha_{i}(p)$.

A parabolic structure at $p$ is called trivial if $l_{p}(p)=1$.

A parabolic vector bundle $E_{*}$ on $X$ is a vector bundle $E$ on $X$ together with a parabolic structure at each $p \in P$.

We shall fix $P$. We also assume that the parabolic weights are rational, hence there is an integer $N$ such that the weights are integral multiples of $1 / N$. Let

$$
w t_{p}(E)=\sum_{i=1}^{l_{p}} n_{i}(p) \alpha_{i}(p)
$$

and $w t(E)=\sum_{p \in P} w t\left(E_{p}\right)$. The (parabolic) degree and slope of $E_{*}$ are defined by

$$
d\left(E_{*}\right)=d(E)+w t(E), \mu\left(E_{*}\right)=d\left(E_{*}\right) / r(E)
$$

(Semi)stability of parabolic bundles is defined using parabolic slope. See [MeS] for the details.

Let $M(r, d)$ denote the moduli space parametrizing all stable parabolic vector bundles with rank, degree and parabolic structure fixed. It is an irreducible smooth quasi-projective variety with

$$
\operatorname{dim} M(r, d)=r^{2}(g-1)+1+\sum_{p} \sum_{i>j} n_{i}(p) n_{j}(p) .
$$

DeFINITION 2.2. A parabolic homomorphism (respectively, strongly parabolic homomorphism) of parabolic bundles

$$
f: E_{*} \longrightarrow F_{*}
$$

is a homomorphism of vector bundles $f: E \rightarrow F$ such that if $\left\{\gamma_{i}(p)\right\}$ and $\left\{\beta_{j}(p)\right\}$ are weights at $p \in P$ of $E$ and $F$ respectively, then $f\left(E_{p, i}\right) \subset F_{p, j+1}$ if $\gamma_{i}(p)>\beta_{j}(p)$ (respectively, $\gamma_{i}(p) \geq \beta_{j}(p)$ ).

Let $\operatorname{Hom}\left(E_{*}, F_{*}\right)$ (respectively, $\left.\operatorname{Hom}_{s t}\left(E_{*}, F_{*}\right)\right)$ denote the vector space of parabolic (respectively, strongly parabolic) homomorphisms from $E_{*}$ to $F_{*}$. by

DEFINITION 2.3. The sheaf of parabolic homomorphisms from $E_{*}$ to $F_{*}$ is defined

$$
\operatorname{Hom}\left(E_{*}, F_{*}\right)(U):=\left\{\operatorname{Hom}\left(\left.\left.E_{*}\right|_{U} \longrightarrow F_{*}\right|_{U}\right)\right\}
$$


for any open set $U \subset X$. The sheaf of strongly parabolic homomorphisms $\operatorname{Hom}_{s t}\left(E_{*}, F_{*}\right)$ is defined similarly.

Therefore, there are short exact sequences

$$
\begin{gathered}
0 \longrightarrow \operatorname{Hom}\left(E_{*}, F_{*}\right) \longrightarrow \operatorname{Hom}(E, F) \longrightarrow T_{E, F} \longrightarrow 0 \\
0 \longrightarrow \operatorname{Hom}_{s t}\left(E_{*}, F_{*}\right) \longrightarrow \operatorname{Hom}\left(E_{*}, F_{*}\right) \longrightarrow T_{E, F}^{0} \longrightarrow 0 .
\end{gathered}
$$

Here $T_{E, F}$ and $T_{E, F}^{0}$ are torsion sheaves supported on $P$ with

$$
\operatorname{dim} T_{E, F}=\sum_{p \in P} \sum_{\gamma_{i}(p)>\beta_{j}(p)} n_{i}^{E}(p) n_{j}^{F}(p)
$$

and

$$
\operatorname{dim} T_{E, F}^{0}=\sum_{p \in P} \sum_{\gamma_{i}(p)=\beta_{j}(p)} n_{i}^{E}(p) n_{j}^{F}(p) .
$$

Define rational numbers $t_{E, F}$ and $t_{E, F}^{0}$ by

$$
r(E) r(F) t_{E, F}=\operatorname{dim} T_{E, F} \text { and } r(E) r(F) t_{E, F}^{0}=\operatorname{dim} T_{E, F}^{0} .
$$

For $p \in P$, let $W_{E}(p)$ and $W_{F}(p)$ denote the sets of weights of $E_{*}$ and $F_{*}$ respectively, and set $W(p)=W_{E}(p) \cup W_{F}(p)$. Arrange the elements of $W(p)$ in a sequence

$$
0 \leq \alpha_{1}(p)<\alpha_{2}(p)<\cdots<\alpha_{\# W(p)}(p)<1 .
$$

In particular, $\alpha_{i}(p)=\gamma_{j}(p)$ or $\alpha_{i}(p)=\beta_{k}(p)$ for some $j, k$. For any $\alpha_{i}(p) \in W(p)$, we again denote by $n_{i}^{E}(p)$ the multiplicity of $\alpha_{i}(p)$ in the fiber $E_{p}$, with the convention that $n_{i}^{E}(p)=0$ if $\alpha_{i}$ is not a weight of $E$ at $p$. It is easy to check that

$$
r(E) r(F) t_{E, F}=\sum_{p \in P} \sum_{i>j} n_{i}^{E}(p) n_{j}^{F}(p)
$$

and

$$
r(E) r(F) t_{E, F}^{0}=\sum_{p \in P} \sum_{i} n_{i}^{E}(p) n_{i}^{F}(p) .
$$

More generally, we have the following lemma.

Lemma 2.4. Consider an exact sequence of parabolic vector bundles

$$
0 \longrightarrow E_{*}^{\prime} \longrightarrow E_{*} \longrightarrow E_{*}^{\prime \prime} \longrightarrow 0,
$$

with $E_{*}, E_{*}^{\prime}$ and $E_{*}^{\prime \prime}$ of ranks $r, r^{\prime}$ and $r^{\prime \prime}$ respectively. If $F_{*}$ is any parabolic vector bundle, then

1. $r^{2} t_{E, E}={r^{\prime}}^{2} t_{E^{\prime}, E^{\prime}}+r^{\prime \prime 2} t_{E^{\prime \prime}, E^{\prime \prime}}+r^{\prime} r^{\prime \prime}\left(t_{E^{\prime}, E^{\prime \prime}}+t_{E^{\prime \prime}, E^{\prime}}\right)$,

2. $r^{\prime} t_{E^{\prime}, F}+r^{\prime \prime} t_{E^{\prime \prime}, F}=r t_{E, F}$,

3. $r^{\prime} t_{F, E^{\prime}}+r^{\prime \prime} t_{F, E^{\prime \prime}}=r t_{F, E}$, ,

4. $p_{0}=t_{E, F}+t_{F, E}+t_{E, F}^{0}\left(\right.$ recall that $\left.p_{0}:=\# P\right)$. 
Proof. (1) We have $n_{i}^{E}(p)=n_{i}^{E^{\prime}}(p)+n_{i}^{E^{\prime \prime}}(p)$ for all $i$. Substituting this in the left hand side of (1) and expanding terms, we get the result.

(2) We have $r^{\prime} r(F) t_{E, F}=\sum_{p \in P} \sum_{i>j} n_{i}^{E}(p) n_{j}^{F}(p)$. The result follows easily by substituting for $n_{i}^{E}(p)$ as in the proof of (1).

(3) This follows exactly as (2).

(4) Note that $r(E)=\sum_{i} n_{i}^{E}(p)$ for each $p \in P$. Hence

$r(E) r(F)=\sum_{i, j} n_{i}^{E}(p) n_{j}^{F}(p)=\sum_{i>j} n_{i}^{E}(p) n_{j}^{F}(p)+\sum_{j>i} n_{i}^{E}(p) n_{j}^{F}(p)+\sum_{i} n_{i}^{E}(p) n_{i}^{F}(p)$.

The result follows by summing over $p \in P$. $\square$

Lemma 2.5. Let $B_{*}$ and $C_{*}$ be parabolic vector bundles.

1. $r(B) w t_{p}(C)-r(C) w t_{p}(B)+\sum_{i>j} n_{i}^{B}(p) n_{j}^{C}(p) \geq 0$ for each $p \in P$, and equality holds if and only if the union of weights of $B$ and $C$ (at $p$ ) is a singleton set.

2. $r(B) w t(C)-r(C) w t(B)+r(B) r(C) t_{B, C} \geq 0$ with the equality holding if and only if the union of weights of $B$ and $C$ at each $p \in P$ is a singleton set.

3. $\chi\left(\operatorname{Hom}\left(B_{*}, C_{*}\right)\right)=r(B) d(C)-r(C) d(B)+r(B) r(C)\left(1-g-t_{B, C}\right)$.

Proof. (1) Since we are fixing $p$, we shall omit $(p)$ in the calculations in this proof. Note that $r(B)=\sum_{i} n_{i}^{B}$ and $r(C)=\sum_{j} n_{j}^{C}$. Hence the left hand side of (1) is

$$
\begin{gathered}
\sum_{i, j} n_{i}^{B} n_{j}^{C} \alpha_{j}-\sum_{i, j} n_{j}^{C} n_{i}^{B} \alpha_{i}+\sum_{i>j} n_{i}^{B} n_{j}^{C}=\sum_{i, j} n_{i}^{B} n_{j}^{C}\left(\alpha_{j}-\alpha_{i}\right)+\sum_{i>j} n_{i}^{B} n_{j}^{C} \\
=\sum_{i \leq j} n_{i}^{B} n_{j}^{C}\left(\alpha_{j}-\alpha_{i}\right)+\sum_{i>j}\left(1+\alpha_{j}-\alpha_{i}\right) n_{i}^{B} n_{j}^{C} .
\end{gathered}
$$

Since $\alpha_{j} \geq \alpha_{i}$ (respectively, $\alpha_{j}>\alpha_{i}$ ) for $j \geq i$ (respectively, $j>i$ ), the first summation is nonnegative and it is zero if and only if $n_{i}^{B} n_{j}^{C}=0 \forall i<j$. Since $1-\alpha_{i}>0 \forall i$, the second summation is nonnegative and it is zero if and only if $n_{i}^{B} n_{j}^{C}=0 \forall i>j$. The result now follows.

The part (2) follows from part (1) by summing over $p \in P$.

Part (3) follows easily from the exact sequence Eqn. (2.1).

Definition 2.6. A parabolic vector bundle $E_{*}$ is $(l, m)$-stable if for every proper subbundle $F_{*}$ of $E_{*}$, the inequality

$$
\frac{d\left(F_{*}\right)+l}{r(F)}<\frac{d\left(E_{*} / F_{*}\right)-m}{r(E / F)}
$$

holds.

Clearly an $(l, m)$-stable parabolic bundle is $(0,0)$-stable (i.e., stable in the usual sense) if $l, m \geq 0$. Let $x \in X$ be a closed point with $x \notin P$. Let

$$
0 \longrightarrow E_{*}^{\prime} \longrightarrow E_{*} \longrightarrow \mathbb{C}_{x} \longrightarrow 0
$$

be an exact sequence of coherent sheaves and the parabolic structure on $E_{*}^{\prime}$ is the one induced by $E_{*}$ using the inclusion map, that is, $E_{*}^{\prime}$ is obtained from $E_{*}$ by an elementary transformation. It is easy to see from the above definition that if $E_{*}$ is 
$(l, m)$-stable, then $E_{*}^{\prime}$ is $(l, m-1)$-stable and if $E_{*}^{\prime}$ is $(l, m)$-stable then $E_{*}$ is $(l-1, m)$ stable.

Proposition 2.7. (1) Assume that the fixed parabolic structure on elements in $M(r, d)$ is not trivial at some parabolic point $p$. Then the $(0,1)$-stable parabolic bundles form a nonempty Zariski open subset of the moduli space $M(r, d)$ for $g \geq 2$.

If the parabolic structure is trivial at each parabolic point, then the above assertion holds for $g \geq 3$.

(2) The statement in (1) holds when $(0,1)$-stable bundles are replaced by $(1,0)$ stable bundles.

Proof. (1) A stable parabolic bundle $E_{*}$ fails to be $(0,1)$-stable if it has a subbundle $E_{*}^{\prime}$ of rank $r^{\prime}$, degree $d^{\prime}$ such that

$$
\frac{d^{\prime}+w t E^{\prime}}{r^{\prime}} \geq \frac{d^{\prime \prime}+w t E^{\prime \prime}-1}{r^{\prime \prime}} .
$$

Hence the ranks $r^{\prime \prime}$, degrees $d^{\prime \prime}$ and weight-multiplicities $n_{i, p}^{\prime \prime}$ for quotients $E_{*}^{\prime \prime}$ of elements of $M(r, d)$ vary over finite sets. Using the properness of (parabolic) Quot schemes, it follows that the complement of the subset consisting of $(0,1)$-stable bundles in $M(r, d)$ is a finite union of closed sets. Thus the $(0,1)$-stable bundles form an open set in $M(r, d)$.

Let $h^{1}:=h^{1}\left(\operatorname{Hom}\left(E_{*}^{\prime \prime}, E_{*}^{\prime}\right)\right)$. Since $E_{*}$ is stable, $h^{0}\left(\operatorname{Hom}\left(E_{*}^{\prime \prime}, E_{*}^{\prime}\right)\right)=0$. Hence

$$
h^{1}=-\chi\left(\operatorname{Hom}\left(E_{*}^{\prime \prime}, E_{*}^{\prime}\right)\right)=r^{\prime} d^{\prime \prime}-r^{\prime \prime} d^{\prime}+r^{\prime} r^{\prime \prime}\left(g-1+t_{E^{\prime \prime}, E^{\prime}}\right)
$$

by Lemma 2.5(3). The above inequality Eqn. (2.4) is equivalent to

$$
r^{\prime} d^{\prime \prime}-r^{\prime \prime} d^{\prime} \leq r^{\prime \prime} w t E^{\prime}-r^{\prime} w t E^{\prime \prime}+r^{\prime} .
$$

Hence $h^{1} \leq r^{\prime} r^{\prime \prime}\left(g-1+t_{E^{\prime \prime}, E^{\prime}}\right)+r^{\prime \prime} w t E^{\prime}-r^{\prime} w t E^{\prime \prime}+r^{\prime}$. The corresponding component of the subvariety of non- $(0,1)$-stable bundles in $M(r, d)$ has dimension $\delta$, then

$$
\begin{gathered}
\delta \leq \operatorname{dim} M\left(r^{\prime}, d^{\prime}\right)+\operatorname{dim} M\left(r^{\prime \prime}, d^{\prime \prime}\right)+h^{1}-1 \\
=r^{2}(g-1)+1+r^{2} t_{E, E}-r^{\prime} r^{\prime \prime}(g-1)+r^{\prime}-r^{\prime} r^{\prime \prime} t_{E^{\prime}, E^{\prime \prime}}+r^{\prime \prime} w t E^{\prime}-r^{\prime} w t E^{\prime \prime}
\end{gathered}
$$

Thus $\delta \leq \operatorname{dim} M(r, d)-r^{\prime} r^{\prime \prime}(g-1)+r^{\prime}$ and the equality holds if and only if $E_{*}$ has all weights equal at all $p \in P$ (Lemma 2.5(2)). Since $r^{\prime \prime}(g-1)-1 \geq 0$ for $g \geq 2$, it follows that $\delta<\operatorname{dim} M(r, d)$ for $g \geq 2$ if $E_{*}$ does not have all weights equal at some $p \in P$. Otherwise, $\delta<\operatorname{dim} M(r, d)$ for $r^{\prime \prime}(g-1)-1>0$ which holds if $g \geq 3$. This completes the proof of (1). We remark that all weights equal at $p$ is equivalent to trivial parabolic structure at $p$.

(2) The proof is similar to that of (1). A stable bundle $E_{*}$ fails to be $(1,0)$-stable if and only if it has a subbundle $E_{*}^{\prime}$ with $r^{\prime} d^{\prime \prime}-r^{\prime \prime} d^{\prime} \leq r^{\prime \prime} w t E^{\prime}-r^{\prime} w t E^{\prime \prime}+r^{\prime \prime}$. The corresponding component of the variety of non- $(1,0)$-stable bundles in $M(r, d)$ has dimension $\delta \leq \operatorname{dim} M(r, d)-r^{\prime} r^{\prime \prime}(g-1)+r^{\prime \prime}+\left(r^{\prime \prime} w t E^{\prime}-r^{\prime} w t E^{\prime \prime}-r^{\prime} r^{\prime \prime} t_{E^{\prime}, E^{\prime \prime}}\right)$. The result follows as in (1). $\mathrm{T}$

The following corollary follows immediately from Proposition 2.7.

COROLlary 2.8. An elementary transformation of a general parabolic bundle $E$ at a nonparabolic point is a general parabolic bundle. The same holds for dual elementary transformations. 
3. Vector bundles with $\Gamma$-action. The main results of this section are the following. (1): an analog for vector bundles with finite group actions of the result of Mukai and Sakai in [MS] giving lower bound on the degree of maximal subbundles of a vector bundle; (2): Yoneda pairing for vector bundles with the action of a finite group; and (3): corresponding results for parabolic vector bundles. In fact, here we prove the analogue of Mukai-Sakai bound for parabolic $G$-bundles, where $G$ is any complex reductive algebraic group.

Definition 3.1. Let $Y$ be a connected smooth projective curve over $\mathbb{C}$ and $\Gamma$ a finite subgroup of the automorphism group of $Y$. A vector bundle $E^{\prime}$ on $Y$ with $\Gamma$-action is a vector bundle $E^{\prime}$ on $Y$ with an action of $\Gamma$ on $E^{\prime}$ as automorphisms of vector bundle over the action of $\Gamma$ on $Y$. In other words, the action of any $\gamma \in \Gamma$ on $E^{\prime}$ is an automorphism of the vector bundle $E^{\prime}$ over the automorphism of $Y$ defined by $\gamma$.

A vector bundle $E^{\prime}$ with $\Gamma$-action is called stable if for any $\Gamma$-invariant proper subbundle $F^{\prime} \subset E^{\prime}$ of positive rank the inequality

$$
\frac{\text { degree }\left(F^{\prime}\right)}{\operatorname{rank}\left(F^{\prime}\right)}<\frac{\operatorname{degree}\left(E^{\prime}\right)}{\operatorname{rank}\left(E^{\prime}\right)}
$$

is valid.

Given a vector bundle $E^{\prime}$ over $Y$ with $\Gamma$-action, the direct image $h_{*} E^{\prime}$, where $h: Y \longrightarrow Y / \Gamma$ is the quotient map, is equipped with an action of $\Gamma$ as vector bundle automorphisms. The vector bundle $\left(h_{*} E^{\prime}\right)^{\Gamma}$ over $Y / \Gamma$ defined by the invariant part has a natural parabolic structure. The parabolic structure on $\left(h_{*} E^{\prime}\right)^{\Gamma}$ is defined using the subsheaves

$$
\left(h_{*} E^{\prime} \otimes \mathcal{O}_{Y}(-k D)\right)^{\Gamma} \subset\left(h_{*} E^{\prime}\right)^{\Gamma}
$$

where $k \geq 0$, and $D \subset Y$ is the reduced divisor where $h$ is ramified; see [Bi] for the details of the construction of parabolic structure.

Let $X$ be a complex projective curve and $P \subset X$ a finite set of points. Fix the parabolic weights and their multiplicities over $P$. Then there exists a Galois covering of order $N$

$$
h: Y \longrightarrow X
$$

ramified over $P$ such that all parabolic bundles over $X$ (of the given parabolic type) arise from vector bundles on $Y$ with $\Gamma$-action (of fixed topological type). This gives a bijective correspondence between vector bundles with $\Gamma$-action on $Y$ and parabolic vector bundles on $X[\mathrm{Bi}$. The bijective correspondence preserves (semi)stability [Bi].

We will always fix the rank, degree and the parabolic structure, or equivalently, the topological type of the $\Gamma$-action. Then the moduli spaces of stable parabolic vector bundles, or of stable vector bundles with $\Gamma$-action, are irreducible quasiprojective varieties. By a general bundle we mean a general element of the corresponding moduli space.

The $\Gamma$-action on a vector bundle induces an action of $\Gamma$ on any of its cohomologies. Let $H^{i}\left(Y, E^{\prime}\right)^{\Gamma}$ and $H o m\left(E^{\prime}, F^{\prime}\right)^{\Gamma}$ denote the $\Gamma$-invariants in $H^{i}\left(Y, E^{\prime}\right)$ and $\operatorname{Hom}\left(E^{\prime}, F^{\prime}\right)$ respectively.

We shall need the following known facts (the proofs can be found in [Bi]).

Lemma 3.2. For any vector bundles $E^{\prime}$ and $F^{\prime}$ on $Y$ with $\Gamma$-action and the associated parabolic bundles $E_{*}$ and $F_{*}$ on $X=Y / \Gamma$ 
1. $d\left(E^{\prime}\right)=N d\left(E_{*}\right), N=|\Gamma|$, and $r\left(E^{\prime}\right)=r\left(E_{*}\right)$;

2. $H^{i}\left(Y, E^{\prime}\right)^{\Gamma} \cong H^{i}(X, E)$, where $E$ is the vector bundle underlying the parabolic bundle $E_{*}$;

3. $\left(h_{*} \operatorname{Hom}\left(E^{\prime}, F^{\prime}\right)\right)^{\Gamma} \cong \operatorname{Hom}\left(E_{*}, F_{*}\right)$.

Proposition 3.3. (1) Let $E^{\prime}$ and $F^{\prime}$ be general stable vector bundles with $\Gamma$ action. Consider the pairing

$$
\varphi: H^{1}\left(\operatorname{End}\left(E^{\prime}\right)\right)^{\Gamma} \longrightarrow \operatorname{Hom}\left(\left(H^{0}\left(E^{\prime *} \otimes F^{\prime}\right)\right)^{\Gamma},\left(H^{1}\left(E^{\prime *} \otimes F^{\prime}\right)\right)^{\Gamma}\right)
$$

defined by $\varphi(\xi)(\alpha)=\xi \cup \alpha$ for any $\xi \in H^{1}\left(\operatorname{End}\left(E^{\prime}\right)\right)^{\Gamma}$ and $\alpha \in H^{0}\left(E^{\prime *} \otimes F^{\prime}\right)^{\Gamma}$. Then this pairing is identically zero.

(2) Let $E_{*}$ and $F_{*}$ be general stable parabolic vector bundles. Then the cup product pairing

$$
H^{1}\left(\operatorname{Hom}\left(E_{*}, E_{*}\right)\right) \otimes H^{0}\left(\operatorname{Hom}\left(E_{*}, F_{*}\right)\right) \longrightarrow H^{1}\left(\operatorname{Hom}\left(E_{*}, F_{*}\right)\right)
$$

is identically zero.

(3) Let the assumptions be as in (2). Then the pairing of vector spaces (defined by composition of homomorphisms)

$$
\operatorname{Hom}\left(E_{*}, F_{*}\right) \times \operatorname{Hom}_{s t}\left(F_{*}, E_{*} \otimes K(P)\right) \longrightarrow \operatorname{Hom}_{s t}\left(E_{*}, E_{*} \otimes K(P)\right)
$$

is identically zero. Here $K$ denotes the canonical bundle of $X$.

Proof. Let $T=\operatorname{Spec} k[\epsilon] / \epsilon^{2}$. The closed point of $T$ will be denoted by $t_{0}$. Let

$$
p_{Y}: Y \times T \longrightarrow Y
$$

be the natural projection. Let $\mathcal{E} \longrightarrow Y \times T$ be a versal deformation, where $\mathcal{E}$ is a family of vector bundles with $\Gamma$-action such that $\mathcal{E}_{\mid Y \times t_{0}} \cong E^{\prime}$. Define $\mathcal{F}:=$ $\left(\mathcal{E}^{*} \otimes p_{Y}^{*} F^{\prime}\right)$. One has the exact sequence

$$
0 \longrightarrow \epsilon \mathcal{F} \longrightarrow \mathcal{F} \longrightarrow \epsilon \mathcal{F}=\mathcal{F} \bmod \epsilon \longrightarrow 0,
$$

where the projection is given by multiplication by $\epsilon$. Taking $p_{Y *}$ gives the exact sequence

$$
0 \longrightarrow V_{0} \longrightarrow V \longrightarrow V_{0} \longrightarrow 0
$$

on $Y$, where $V:=\left(p_{Y}\right)_{*}(\mathcal{F}), V_{0}:=E^{\prime *} \otimes F^{\prime}$ and $V_{0}^{\prime} \cong V_{0}$. Let $\xi \in H^{1}\left(Y, \operatorname{End}\left(V_{0}\right)\right)^{\Gamma}$ be the element corresponding to this short exact sequence of vector bundles. The above sequence of vector bundles gives the long exact sequence of cohomologies

$$
\cdots \longrightarrow H^{0}(V)^{\Gamma} \longrightarrow H^{0}\left(V_{0}\right)^{\Gamma} \stackrel{\delta}{\longrightarrow} H^{1}\left(V_{0}\right)^{\Gamma} \longrightarrow \cdots
$$

where the connecting homomorphism $\delta$ satisfies the identity $\delta(\alpha)=\xi \cup \alpha$ for all $\alpha \in H^{0}\left(V_{0}\right)^{\Gamma}$. Consequently, $\xi \cup \alpha=0$ if and only if $\alpha$ lifts to an element of $H^{0}(V)^{\Gamma}$. We shall check that the map $H^{0}(V)^{\Gamma} \rightarrow H^{0}\left(V_{0}\right)^{\Gamma}$ is surjective.

The moduli space of stable vector bundles with $\Gamma$-action is reduced and irreducible. Hence there exists a locally complete family $\mathcal{E}^{\prime} \longrightarrow Y \times S$ of stable vector bundles, with $\Gamma$-action, of rank $r$ and degree $d$ parametrized by a variety $S$ such that $\mathcal{E}^{\prime}{ }_{\mid Y \times s_{0}} \cong E^{\prime}$ for some $s_{0} \in S$. The condition that the family is locally complete 
means that the morphism from $S$ to the corresponding moduli space of stable vector bundles with $\Gamma$-action is dominant.

Let $\mathcal{F}^{\prime}=\mathcal{E}^{\prime *} \otimes p_{Y}^{*} F^{\prime}$. Going to an open subset $U$ of $S$ (if necessary), we may assume that $R^{0} p_{U_{*}}\left(\mathcal{F}^{\prime}\right)$ and $R^{1} p_{U_{*}}\left(\mathcal{F}^{\prime}\right)$ are locally free, where $p_{U}: Y \times U \longrightarrow U$ is the natural projection. Then $R^{0} p_{U_{*}}\left(\mathcal{F}^{\prime}\right)$ is compatible with base change. In other words, for all $f: T^{\prime} \rightarrow U$, if $\mathcal{F}^{\prime} T^{\prime}$ is the pull back of $\mathcal{F}^{\prime}$ to $Y \times T^{\prime}$ by the morphism $\operatorname{id}_{Y} \times f$, then one has $\left(p_{T^{\prime}}\right)_{*} \mathcal{F}^{\prime} T^{\prime} \cong f^{*}\left(p_{U_{*}} \mathcal{F}^{\prime}\right)$, where $p_{T^{\prime}}: Y \times T^{\prime} \longrightarrow T^{\prime}$ is the natural projection.

Let $q: T \longrightarrow U$ be a morphism with $q\left(t_{0}\right)=s_{0}$. The above mentioned compatibility condition of $R^{0} p_{U_{*}}\left(\mathcal{F}^{\prime}\right)$ under base change for the morphism $f=q$ says that $p_{T_{*}}(\mathcal{F}) \cong q^{*} p_{U_{*}}\left(\mathcal{F}^{\prime}\right)$. Since the latter is locally free, so is the former. Therefore, we have

$$
H^{0}\left(T, p_{T *} \mathcal{F}\right) \cong H^{0}(Y \times T, \mathcal{F}) \cong H^{0}(Y, V)
$$

where $p_{T}$ is the projection of $Y \times T$ to $T$. On the other hand, the restriction homomorphism

$$
H^{0}\left(T, p_{T *} \mathcal{F}\right) \longrightarrow H^{0}\left(t_{0},\left.\left(p_{T *} \mathcal{F}\right)\right|_{t_{0}}\right) \cong H^{0}\left(Y, \mathcal{F}_{t_{0}}\right)
$$

is surjective (restriction of the sections of a vector bundle over $T$ to its fiber over $t_{0}$ is surjective). Since the homomorphism

$$
H^{0}(Y, V)^{\Gamma} \cong H^{0}\left(T, p_{T *} \mathcal{F}\right)^{\Gamma} \longrightarrow H^{0}\left(Y, \mathcal{F}_{t_{0}}\right)^{\Gamma}=H^{0}\left(Y, V_{0}\right)^{\Gamma}
$$

obtained from the combination of Eqn. (3.2) and Eqn. (3.3) coincides with the homomorphism

$$
H^{0}(Y, V)^{\Gamma} \longrightarrow H^{0}\left(Y, V_{0}\right)^{\Gamma}
$$

in Eqn. (3.1), we conclude that the homomorphism $H^{0}(Y, V)^{\Gamma} \longrightarrow H^{0}\left(Y, V_{0}\right)^{\Gamma}$ in Eqn. (3.1) is surjective. Thus any $\alpha \in H^{0}\left(V_{0}\right)^{\Gamma}$ lifts to an element of $H^{0}(V)^{\Gamma}$. This completes the proof of part 1.

Proof of part (2): The part (1) says that the cup-product pairing

$$
H^{1}\left(\operatorname{Hom}\left(E^{\prime}, E^{\prime}\right)\right)^{\Gamma} \times H^{0}\left(\operatorname{Hom}\left(E^{\prime}, F^{\prime}\right)\right)^{\Gamma} \rightarrow H^{1}\left(\operatorname{Hom}\left(E^{\prime}, F^{\prime}\right)\right)^{\Gamma}
$$

is zero. Since $H^{i}\left(\operatorname{Hom}\left(E^{\prime}, F^{\prime}\right)\right)^{\Gamma} \cong H^{i}\left(\operatorname{Hom}\left(E_{*}, F_{*}\right)\right)$ (see Lemma 3.2), the result follows.

Proof of part (3): We claim that

$$
\operatorname{Hom}\left(E_{*}, F_{*}\right)^{*} \cong \operatorname{Hom}_{s t}\left(F_{*}, E_{*}(P)\right) .
$$

One has $\operatorname{Hom}(E, F(-P)) \subset \operatorname{Hom}\left(E_{*}, F_{*}\right) \subset \operatorname{Hom}(E, F)$. Dualizing gives $\operatorname{Hom}(F, E) \subset \operatorname{Hom}\left(E_{*}, F_{*}\right)^{*} \subset \operatorname{Hom}(F, E(P))$ with succesive quotients isomorphic to $\mathbb{C}^{r^{\prime} r^{\prime \prime} t_{E, F}}$ and $\mathbb{C}^{q}$, where $q=r^{\prime} r^{\prime \prime}\left(t_{F, E}^{0}+t_{F, E}\right)=r^{\prime} r^{\prime \prime}\left(p_{0}-t_{E, F}\right)$. The claim follows from the commutative diagram 


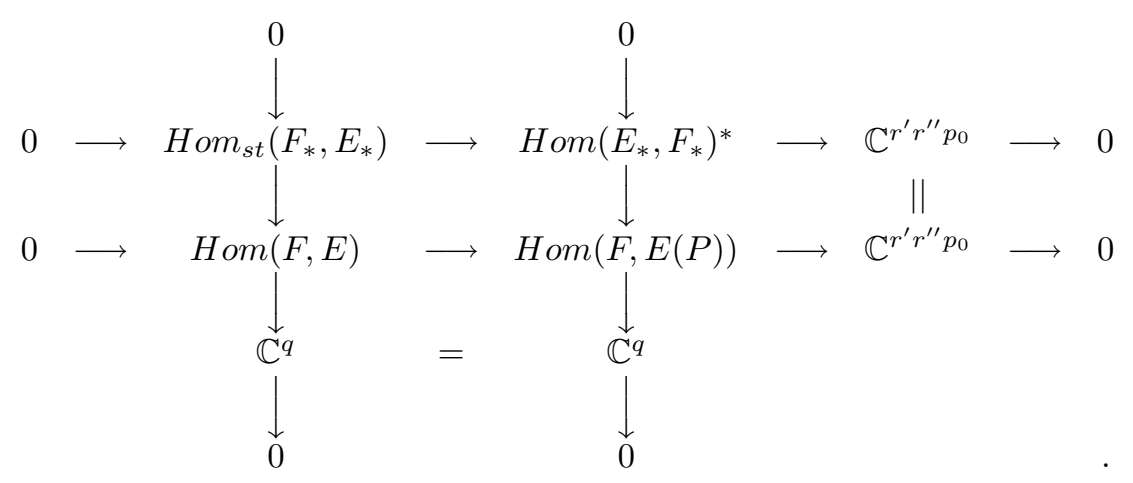

By Serre duality, $H^{1}\left(\operatorname{Hom}\left(E_{*}, F_{*}\right)\right)^{*} \cong H^{0}\left(\left(\operatorname{Hom}\left(E_{*}, F_{*}\right)\right)^{*} \otimes K\right)$. By the claim, the latter is isomorphic to $\operatorname{Hom}_{s t}\left(F_{*}, E_{*} \otimes K(P)\right)$. The result now follows from part (2).

Remark 3.4. Given any integer $\delta$, the general line bundle $L \in \operatorname{Pic}^{\delta}(X)$ has the property that $H^{0}(X, L) \neq 0$ if and only if $\delta \geq g$. Therefore, if $H^{0}(X, L) \neq 0$ for the general line bundle $L \in \operatorname{Pic}^{\delta}(X)$, then $H^{0}\left(X, L^{*} \otimes K\right)=0$ for the general line bundle $L \in \operatorname{Pic}^{\delta}(X)$. This gives a proof of Proposition 3.3(1) when $E^{\prime}$ and $F^{\prime}$ are line bundles with $E^{\prime} \otimes F^{\prime}=K$.

3.1. Parabolic principal bundles. Let $H$ be a connected linear algebraic group over $\mathbb{C}$. Let $E_{H}^{\prime}$ be a connected smooth quasiprojective variety over $k$ and

$$
f: E_{H}^{\prime} \times H \longrightarrow E_{H}^{\prime}
$$

an algebraic right action of $H$ on $E_{H}^{\prime}$.

A parabolic $H$-bundle over $X$ with parabolic structure over $P$ is $\left(E_{H}^{\prime}, f\right)$ as above together with a dominant morphism

$$
\psi: E_{H}^{\prime} \longrightarrow X
$$

satisfying the following conditions:

1. $\psi \circ f=\psi \circ p_{1}$ on $E_{H}^{\prime} \times H$, where $p_{1}$ is the projection of $E_{H}^{\prime} \times H$ to $E_{H}^{\prime}$, that is, the map $\psi$ is equivariant for the action of $H$;

2. for each point $x \in X$, the action of $H$ on the reduced fiber $\psi^{-1}(x)_{\text {red }}$ is transitive;

3. the restriction of $\psi$ to $\psi^{-1}(X \backslash P)$ makes $\psi^{-1}(X \backslash P)$ a principal $H$-bundle over $X \backslash P$, that is, the map $\psi$ is smooth over $\psi^{-1}(X \backslash P)$ and the map to the fiber product

$$
\psi^{-1}(X \backslash P) \times H \longrightarrow \psi^{-1}(X \backslash P) \times_{X \backslash P} \psi^{-1}(X \backslash P)
$$

defined by $(z, g) \longmapsto(z, f(z, g))$ is an isomorphism;

4. for each point $z \in \psi^{-1}(P)_{\text {red }}$, the isotropy at $z$ for the action of $H$ is a finite subgroup of $H$.

See $[\mathrm{BBN}]$ for the details.

For notational convenience, a parabolic $H$-bundle defined as above will be denoted by $E_{*}$. 
A parabolic $\operatorname{GL}(n, \mathbb{C})$-bundle is same as a parabolic vector bundle of rank $n$ with rational parabolic weights. Using the standard action of $\mathrm{GL}(n, \mathbb{C})$ on $\mathbb{C}^{n}$, the principal $\mathrm{GL}(n, \mathbb{C})$-bundle over $X \backslash P$, defined by a parabolic $\mathrm{GL}(n, \mathbb{C})$-bundle $E_{*}$, gives a vector bundle over $X \backslash P$. This vector bundle has a natural extension, which is constructed using $E_{*}$, that carries the parabolic structure of the parabolic vector bundle corresponding to $E_{*}$.

There is a finite Galois covering

$$
h: Y \longrightarrow X
$$

and a $\Gamma$-linearized $G$-bundle $E_{G}$ over $Y$, where $\Gamma$ is the Galois group of the covering $h$, such that $E_{G}$ corresponds to $E_{*}$ (see $[\mathrm{BBN}]$ ). The covering $h$ is ramified over $P$ and for any $x \in P$, the order of ramification is a multiple of the order of the isotropy subgroup for any point in $\psi^{-1}(x)_{\text {red }}$. See [KMM, Ch. 1.1, p. 303-305] for the construction of such a covering.

Let $E_{*}$ be a parabolic $G$-bundle over $X$ with parabolic structure over the divisor $P$. Let $Q$ denote a parabolic subgroup of $G$. A reduction of structure group to $Q$ of a parabolic $G$-bundle $E_{*}$ is a section

$$
\sigma: X \longrightarrow E_{G}^{\prime} / Q
$$

of the natural projection of $E_{G}^{\prime} / Q$ to $X$, where $E_{G}^{\prime}$ is the underlying variety for $E_{*}$. Note that $q^{-1}(\sigma(X))$, where $q$ is the projection of $E_{G}^{\prime}$ to $E_{G}^{\prime} / Q$, is parabolic $Q$-bundle. Conversely, if $E_{Q}^{\prime} \subset E_{G}^{\prime}$ is a parabolic $Q$-bundle, then it defines a section $\sigma$ of $E_{G}^{\prime} / Q$ as above. This section $\sigma$ has the property that $q^{-1}(\sigma(X))$ coincides with $E_{Q}^{\prime}$.

We noted above that there is a $\Gamma$-linearized principal $G$-bundle $E_{G}$ over $Y$ that corresponds to the parabolic $G$-bundle $E_{*}$ over $X$. Moreover, the reductions of $E_{*}$ to $Q$ are in bijective correspondence with the $\Gamma$-invariant reductions of $E_{G}$ to $Q$ (see $[\mathrm{BBN}])$.

Consider the $Q$-module $\mathfrak{g} / \mathfrak{q}$, where $\mathfrak{g}$ and $\mathfrak{q}$ are the Lie algebras of $G$ and $Q$ respectively. For a reduction $E_{Q}^{\prime} \subset E_{G}^{\prime}$ of a parabolic $G$-bundle $E_{*}$ to the parabolic subgroup $Q$, the pair $\left(E_{Q}^{\prime}, \mathfrak{g} / \mathfrak{q}\right)$ defines a parabolic vector bundle $E_{Q}^{\prime}(\mathfrak{g} / \mathfrak{q})$ over $X$. Note that $E_{Q}^{\prime}(\mathfrak{g} / \mathfrak{q})$ is a quotient of the parabolic vector bundle $E_{*}(\mathfrak{g})$ associated to $E_{*}$ for the adjoint representation of $G$ on $\mathfrak{g}$. Therefore, there is a constant $N\left(E_{*}\right) \in \mathbb{Z}$ such that the parabolic degree

$$
\operatorname{par}-\operatorname{deg}\left(E_{Q}^{\prime}(\mathfrak{g} / \mathfrak{q})\right) \geq N\left(E_{*}\right)
$$

for every reduction $E_{Q}^{\prime} \subset E_{G}^{\prime}$ to $Q$. Let

$$
c\left(E_{*}\right) \in \mathbb{Z}
$$

be the smallest value of $\operatorname{par}-\operatorname{deg}\left(E_{Q}^{\prime}(\mathfrak{g} / \mathfrak{q})\right)$ taken over all reductions of $E_{*}$ to $Q$.

Proposition 3.5. Let $E_{Q}^{\prime} \subset E_{G}^{\prime}$ be a reduction to $Q$ of the parabolic $G$-bundle $E_{G}^{\prime}$ to a parabolic subgroup $Q \subset G$ such that par- $\operatorname{deg}\left(E_{Q}^{\prime}(\mathfrak{g} / \mathfrak{q})\right)=c\left(E_{*}\right)$. Then

$$
\operatorname{degree}\left(E_{Q}^{\prime}(\mathfrak{g} / \mathfrak{q})\right) \leq g_{X} \cdot \operatorname{dim} G / Q
$$

where $g_{X}$ is the genus of $X$.

Proof. Let $E_{G}$ be the $\Gamma$-linearized $G$-bundle over $Y$ corresponding to the parabolic $G$-bundle $E_{G}^{\prime}$. In the earlier mentioned bijective correspondence between $\Gamma$ invariant reductions of $E_{G}$ to $Q$ and reductions of $E_{G}^{\prime}$ to $Q$, we have

$$
\# \Gamma \cdot \operatorname{par}-\operatorname{deg}\left(E_{Q}^{\prime}(\mathfrak{g} / \mathfrak{q})\right)=\operatorname{degree}\left(\operatorname{ad}\left(E_{G}\right) / \operatorname{ad}\left(E_{Q}\right)\right),
$$


where $E_{Q}$ is the reduction of $E_{G}$ corresponding to the reduction $E_{Q}^{\prime}$ of $E_{G}^{\prime}$. So, if $E_{Q}^{\prime} \subset E_{G}^{\prime}$ is a reduction such that par-deg $\left(E_{Q}^{\prime}(\mathfrak{g} / \mathfrak{q})\right)=c\left(E_{*}\right)$, then the corresponding reduction $E_{Q} \subset E_{G}$ has the property that degree $\left(\operatorname{ad}\left(E_{G}\right) / \operatorname{ad}\left(E_{Q}\right)\right)=c\left(E_{G}\right)$, where $c\left(E_{G}\right)$ is the smallest value of degree $\left(\operatorname{ad}\left(E_{G}\right) / \operatorname{ad}\left(E_{Q}\right)\right)$ taken over $\Gamma$-invariant reductions of $E_{G}$ to $Q$.

The vector bundle $\left(h_{*}\left(\operatorname{ad}\left(E_{G}\right) / \operatorname{ad}\left(E_{Q}\right)\right)\right)^{\Gamma}$ over $X$ defined by the invariant direct image is identified with the underlying vector bundle of the parabolic vector bundle $E_{Q}^{\prime}(\mathfrak{g} / \mathfrak{q})[\mathrm{BBN}]$. Consequently, we have

$$
H^{i}\left(Y, \operatorname{ad}\left(E_{G}\right) / \operatorname{ad}\left(E_{Q}\right)\right)^{\Gamma} \cong H^{i}\left(X, E_{Q}^{\prime}(\mathfrak{g} / \mathfrak{q})\right) .
$$

Now [BB, Proposition 3.1] and the Riemann-Roch for the underlying vector bundle for $E_{Q}^{\prime}(\mathfrak{g} / \mathfrak{q})$ give

$$
\begin{aligned}
\operatorname{dim} G / Q & \geq \sum_{i=0}^{1}(-1)^{i} \operatorname{dim} H^{i}\left(Y, \operatorname{ad}\left(E_{G}\right) / \operatorname{ad}\left(E_{Q}\right)\right)^{\Gamma} \\
& =\operatorname{degree}\left(E_{P}^{\prime}(\mathfrak{g} / \mathfrak{q})\right)+\left(1-g_{X}\right) \operatorname{dim} G / Q .
\end{aligned}
$$

So, $g_{X} \cdot \operatorname{dim} G / Q \geq \operatorname{degree}\left(E_{Q}^{\prime}(\mathfrak{g} / \mathfrak{q})\right)$, and the proof of the proposition is complete.

Definition 3.6. Let $E^{\prime}$ be a vector bundle on $Y$ with $\Gamma$-action. A nonzero proper $\Gamma$-invariant subbundle $F^{\prime} \subset E^{\prime}$ is said to be $\Gamma$-maximal if it has the maximal degree among all $\Gamma$-invariant subbundles of $E^{\prime}$ of the same rank.

Proposition 3.7. Let $F^{\prime}$ be a $\Gamma$-maximal subbundle of a vector bundle $E^{\prime}$ with $\Gamma$-action. Let $F_{*}$ and $E_{*}$ be the parabolic vector bundles corresponding to $F^{\prime}$ and $E^{\prime}$ respectively. Then $F_{*}$ is a maximal parabolic subbundle of $E_{*}$. Also,

1. $\mu_{E / F}-\mu_{F} \leq g+t_{F, E / F}$, and

2. $\mu_{E^{\prime} / F^{\prime}}-\mu_{F^{\prime}} \leq N\left(g+t_{F, E / F}+w t(E / F) / r(E / F)-w t(F) / r(F)\right)$.

Proof. That $F_{*}$ is maximal follows form the first assertion in Lemma 3.2. Also, note that (2) is equivalent to (1) by Lemma 3.2(1).

The part (1) is a particular case of Proposition 3.5. In the notation of Proposition 3.5, taking $G=\operatorname{GL}(r, \mathbb{C})$ and $Q$ the parabolic subgroup determined by the flag $\mathbb{C}^{r_{1}} \subset$ $\mathbb{C}^{r}$, where $r_{1}=r(F)$, one has

$$
\operatorname{degree}\left(\operatorname{Hom}\left(F_{*},(E / F)_{*}\right)\right)=\operatorname{degree}\left(E_{Q}(\mathfrak{g} / \mathfrak{q})\right) \leq g \cdot r(F) r(E / F) .
$$

Here $E$ is the parabolic principal $\mathrm{GL}(r, \mathbb{C})$-bundle corresponding to $E_{*}$, and $\mathfrak{g}$ and $\mathfrak{q}$ are the Lie algebras of $G$ and $Q$ respectively. The statement (1) follows using the fact that $d\left(\operatorname{Hom}\left(F_{*},(E / F)_{*}\right)=r(F) r(E / F)\left(\mu_{E / F}-\mu_{F}-t_{F, E / F}\right)\right.$.

4. General parabolic vector bundles. As before, let $M(r, d)$ be the moduli space of all stable parabolic vector bundles over $X$ of $\operatorname{rank} r$, degree $d$ and fixed rational parabolic weights of fixed multiplicities at finitely many distinct closed points $P \subset X$.

Proposition 4.1. Consider an exact sequence of the form

$$
0 \longrightarrow C_{*} \longrightarrow E_{*} \longrightarrow B_{*} \longrightarrow 0
$$

with $C_{*}, E_{*}$ and $B_{*}$ parabolic vector bundles of ranks $k, r$ and $r-k$ with slopes $\mu_{C}$, $\mu$ and $\mu_{B}$ respectively, and with fixed given parabolic structures. Assume that $E_{*}$ is in $M(r, d)$, in particular, it is stable. Then one has the following. 
1. A general $E_{*} \in M(r, d)$ does not occur in an exact sequence of the form Eqn. (4.1) with $\mu_{B}-\mu_{C}<g-1+t_{C, B}$.

2. Let $\mu_{B}-\mu_{C}=g-1+t_{C, B}$. Then a general parabolic bundle $E_{*}$ has only finitely many (saturated) subbundles $C_{*}$ of rank $k$, slope $\mu_{C}$ and having a given induced parabolic structure. Moreover these subbundles $C_{*}$ and corresponding quotients $B_{*}$ must be general stable parabolic vector bundles in $M\left(k, k \mu_{C}\right)$ and $M\left(r-k,(r-k) \mu_{B}\right)$ respectively.

Proof. (1) The extensions of the form as in Eqn. (4.1) are parametrized by the projective space $\mathbb{P}\left(H^{1}\left(\operatorname{Hom}\left(B_{*}, C_{*}\right)\right)\right.$ where $\operatorname{Hom}\left(B_{*}, C_{*}\right)$ is the sheaf of parabolic homomorphisms. Since stable parabolic bundles are simple, it is easy to see that the stability of $E_{*}$ implies that $H^{0}\left(\operatorname{Hom}\left(B_{*}, C_{*}\right)\right)=0$. By Riemann-Roch theorem and Lemma 2.5(3), one then has

$$
\operatorname{dim} H^{1}\left(B_{*}, C_{*}\right)=k(r-k)\left(g-1+\mu_{B}-\mu_{C}+t_{B, C}\right) .
$$

Let $\delta$ be the dimension of the space of extensions of type Eqn. (4.1) with $B_{*}, C_{*}$ varying over parabolic bundles of the fixed type. Then

$$
\delta \leq \operatorname{dim} M(r-k, d(B))+\operatorname{dim} M(k, d(C))+\operatorname{dim} \mathbb{P} H^{1}\left(\operatorname{Hom}\left(B_{*}, C_{*}\right)\right) .
$$

One has

$$
\operatorname{dim} M(r-k, d(B))=(r-k)^{2}(g-1)+1+(r-k)^{2} t_{B, B}
$$

and $\operatorname{dim} M(k, d(C))=k^{2}(g-1)+1+k^{2} t_{C, C}$. Hence

$$
\delta \leq \operatorname{dim} M(r, d)=r^{2}(g-1)+1+r^{2} t_{E, E}
$$

(respectively, $\left.\delta<\operatorname{dim} M(r, d)=r^{2}(g-1)+1+r^{2} t_{E, E}\right)$ if one has

$$
k(r-k)\left(\mu_{B}-\mu_{C}-(g-1)\right) \leq r^{2} t_{E, E}-(r-k)^{2} t_{B, B}-k^{2} t_{C, C}-k(r-k) t_{B, C}
$$

(respectively, $\left.k(r-k)\left(\mu_{B}-\mu_{C}-(g-1)\right)<r^{2} t_{E, E}-(r-k)^{2} t_{B, B}-k^{2} t_{C, C}-k(r-k) t_{B, C}\right)$. It follows from Lemma 2.4(1) that $\delta \leq \operatorname{dim} M(r, d)$ (respectively, $\delta<\operatorname{dim} M(r, d)$ ) if $\mu_{B}-\mu_{C} \leq g-1+t_{C, B}$ (respectively, $\mu_{B}-\mu_{C}<g-1+t_{C, B}$ ). Thus, for a general $E_{*} \in M(r, d)$, one must have $\mu_{B}-\mu_{C} \geq g-1+t_{C, B}$. This proves (1).

(2) If $B_{*}$ (or $\left.C_{*}\right)$ is not general in $M(r-k, d(B))$ (respectively $M(k, d(C))$ ), then it is determined by parameters whose number is strictly less than $\operatorname{dim} M(r-k, d(B))$ (respectively $M(k, d(C))$ ). Consequently, $\delta<r^{2}(g-1)+1+r^{2} t_{E, E}$. Hence for a general $E_{*}$, both $B_{*}$ and $C_{*}$ must be general stable parabolic vector bundles. Moreover, if $E_{*}$ has infinitely many such subbundles $C_{*}$ then again $E_{*}$ will be determined by the number of parameters which is strictly less than $\delta \leq r^{2}(g-1)+1+t_{E, E}$ and hence cannot be general. Thus $E_{*}$ has only finitely many subbundles $C_{*}$.

Theorem 4.2. Let $A_{*}$ and $B_{*}$ be general stable parabolic vector bundles. Then

$$
\begin{array}{lccc}
\text { (A) }(1): & H^{0}\left(\operatorname{Hom}\left(A_{*}, B_{*}\right)\right)=0 & \text { if } & \mu_{B}-\mu_{A} \leq g-1+t_{A, B}, \\
\text { (A) }(2): & H^{1}\left(\operatorname{Hom}_{*}\left(A_{*}, B_{*}\right)\right)=0 & \text { if } & \mu_{B}-\mu_{A} \geq g-1+t_{A, B}, \\
\text { (B) (1): } & H^{0}\left(\operatorname{Hom}_{s t}\left(A_{*}, B_{*}\right)\right)=0 & \text { if } & \mu_{B}-\mu_{A} \leq g-1+t_{A, B}+t_{A, B}^{0}, \\
\text { (B) }(2): & H^{1}\left(\operatorname{Hom}_{s t}\left(A_{*}, B_{*}\right)\right)=0 & \text { if } & \mu_{B}-\mu_{A} \geq g-1+t_{A, B}+t_{A, B}^{0} .
\end{array}
$$

Proof. (A) (1) The short exact sequence

$$
0 \longrightarrow \operatorname{Hom}\left(A_{*}, B_{*}\right) \longrightarrow \operatorname{Hom}(A, B) \longrightarrow T_{A, B} \longrightarrow 0
$$


gives a long exact sequence of cohomologies

$$
\begin{gathered}
0 \longrightarrow H^{0}\left(\operatorname{Hom}\left(A_{*}, B_{*}\right)\right) \longrightarrow H^{0}(\operatorname{Hom}(A, B)) \longrightarrow H^{0}\left(T_{A, B}\right) \\
\longrightarrow H^{1}\left(\operatorname{Hom}\left(A_{*}, B_{*}\right)\right) \longrightarrow H^{1}(\operatorname{Hom}(A, B)) \longrightarrow 0 .
\end{gathered}
$$

Note that the underlying vector bundle of a general parabolic vector bundle is a general vector bundle. Hence if $\mu_{B}-\mu_{A} \geq g-1$ then $H^{1}(\operatorname{Hom}(A, B))=0$ and if $\mu_{B}-\mu_{A} \leq g-1$ then $H^{0}(\operatorname{Hom}(A, B))=0$ ([Hi, Theorem 4.6]). It follows from the sequence Eqn. (4.2) that $H^{0}\left(\operatorname{Hom}\left(A_{*}, B_{*}\right)\right)=0$ for $\mu_{B}-\mu_{A} \leq g-1$. Hence for proving $(\mathrm{A})(1)$ we may assume that

$$
g-1<\mu_{B}-\mu_{A} \leq g-1+t_{A, B} .
$$

Under this assumption, one has $\chi\left(\operatorname{Hom}\left(A_{*}, B_{*}\right)\right) \leq 0$.

Suppose that

$$
H^{0}\left(\operatorname{Hom}\left(A_{*}, B_{*}\right)\right) \neq 0
$$

Then $H^{1}\left(H o m\left(A_{*}, B_{*}\right)\right) \neq 0$, or equivalently, $H^{0}\left(H o m_{s t}\left(B_{*}, A_{*} \otimes K(P)\right) \neq 0\right.$.

Let

$$
f: A_{*} \longrightarrow B_{*}
$$

be a nonzero homomorphism and

$$
h: B_{*} \longrightarrow A_{*} \otimes K(P)
$$

be a nonzero strongly parabolic homomorphism. By Proposition 3.3(3) we have $h \circ f=$ 0 . If $B_{*}$ is a line bundle, then $h$ is a generic injection and $h \circ f=0$ implies that $f=0$, a contradiction. Thus $(\mathrm{A})(1)$ holds if $r(B)=1$.

We shall prove $(A)(1)$ by induction on $r(B)$. Since $(A)(1)$ is valid for $r(B)=1$, we may assume that $r(B) \geq 2$. Let $r_{1}$ and $r_{2}$ be positive integers with $r_{1}+r_{2}=r(B)$. Let $d_{1}$ and $d_{2}$ be integers with $d_{1}+d_{2}=d(B)$. If $\left\{\gamma_{1}, \cdots, \gamma_{r(B)}\right\}$ is the set of all the parabolic weights of $B_{*}$ with $0 \leq \gamma_{1} \leq \cdots \leq \gamma_{r(B)}$, we split this set into two subsets $S_{1}, S_{2}$ of cardinalities $r_{1}, r_{2}$ respectively. Take a general parabolic vector bundle $B_{1 *}$ (respectively, $B_{2 *}$ ) of rank $r_{1}$ and degree $d_{1}$ (respectively, rank $r_{2}$ and degree $d_{2}$ ) and parabolic structure determined by $S_{1}$ (respectively, $S_{2}$ ). Then one has $T_{A, B}=T_{A, B_{1}}+T_{A, B_{2}}$ and hence $r(B) t_{A, B}=r_{1} t_{A, B_{1}}+r_{2} t_{A, B_{2}}$.

Case 1 (i) $\mu_{B}-\mu_{A} \leq g-2+t_{A, B}$.

In this case, the closed interval

$$
\left[d(B)-r_{2}\left(\mu_{A}+g-1+t_{A, B_{2}}\right), r_{1}\left(\mu_{A}+g-1+t_{A, B_{1}}\right)\right]
$$

has length $\geq 1$. Hence there exists an integer $d_{1}$ satisfying

$$
d(B)-r_{2}\left(\mu_{A}+g-1+t_{A, B_{2}}\right) \leq d_{1} \leq r_{1}\left(\mu_{A}+g-1+t_{A, B_{1}}\right) .
$$

The first inequality in Eqn. (4.3) implies that $\mu_{B_{2}}-\mu_{A} \leq g-1+t_{A, B_{2}}$, while the second one implies that $\mu_{B_{1}}-\mu_{A} \leq g-1+t_{A, B_{1}}$. By induction, this gives $H^{0}\left(\operatorname{Hom}\left(A_{*}, B_{1 *}\right)\right)=0$ and $H^{0}\left(\operatorname{Hom}\left(A_{*}, B_{2 *}\right)\right)=0$, and hence $H^{0}\left(\operatorname{Hom}\left(A_{*}, B_{1 *} \oplus\right.\right.$ $\left.\left.B_{2 *}\right)\right)=0$. By semicontinuity of $\operatorname{dim} H^{0}()$ and the fact that any parabolic vector 
bundle (of fixed parabolic type) can be deformed to a stable parabolic vector bundle (of the same type), it follows that $H^{0}\left(\operatorname{Hom}\left(A_{*}, B_{*}\right)\right)=0$ for a general parabolic vector bundle $B_{*}$ in case $1(\mathrm{i})$.

Case 1 (ii) $g-2+t_{A, B}<\mu_{B}-\mu_{A} \leq g-1+t_{A, B}$.

One has $r(A) r(B)\left(g-2+t_{A, B}\right)<r(A) d(B)-r(B) d(A) \leq r(A) r(B)\left(g-1+t_{A, B}\right)$. Since $r(A) d\left(B^{\prime}\right)-r\left(B^{\prime}\right) d(A) \leq r(A) r(B)\left(g-2+t_{A, B^{\prime}}\right)$ for a general parabolic bundle $B_{*}^{\prime}$ with $r\left(B^{\prime}\right)=r(B), d\left(B^{\prime}\right) \leq d(B)-r(B)$ and same parabolic structure as $B$. It follows by Case $1(\mathrm{i})$ that $H^{0}\left(\operatorname{Hom}\left(A_{*}, B_{*}^{\prime}\right)\right)=0$. Suppose that $H^{0}\left(\operatorname{Hom}\left(A_{*}, B_{*}\right)\right) \neq$ 0 . Then, as seen before, there exists a nonzero morphism $f: A_{*} \rightarrow B_{*}$ and a nonzero strongly parabolic homomorphism $h: B_{*} \rightarrow A_{*} \otimes K(P)$.

We claim that $f$ is a generic surjection. If $f$ is not a generic surjection, then we can do elementary transformations on $B_{*}$ at finitely many non-parabolic points to get a parabolic vector bundle $B^{\prime}{ }_{*}$ with image of $f$ contained in $B^{\prime}{ }_{*}$ with $d\left(B^{\prime}\right) \leq d(B)-r(B)$ and having the same parabolic structure as $B_{*}$. Since elementary transformation of a general parabolic bundle is stable (Corollary 2.8), we can get a general stable bundle $B^{\prime}$ with the above properties. Then by Case 1(i) we have $f=0$, a contradiction. Thus we have proved that $f$ is a generic surjection.

By Proposition 3.3(3), $h \circ f=0$. Then the generic surjectivity of $f$ implies that $h=0$, a contradiction. This completes the proof of $(\mathrm{A})(1)$.

(B)(1), (B)(2). We can deduce (B)(2) from $(\mathrm{A})(1)$ as follows. Let $A^{\prime}{ }_{*}, B^{\prime}{ }_{*}$ be general parabolic bundles. One has

$$
\begin{gathered}
\operatorname{deg}\left(\operatorname{Hom}_{s t}\left(B^{\prime}{ }_{*}, A^{\prime}{ }_{*}\right)\right)=\operatorname{deg}\left(A^{\prime}\right) r\left(B^{\prime}\right)-\operatorname{deg}\left(B^{\prime}\right) r\left(A^{\prime}\right)-\operatorname{dim} T_{B^{\prime}, A^{\prime}}-\operatorname{dim} T_{A^{\prime}, B^{\prime}}^{0} \\
=\operatorname{deg}\left(A^{\prime}\right) r\left(B^{\prime}\right)-\operatorname{deg}\left(B^{\prime}\right) r\left(A^{\prime}\right)-r\left(A^{\prime}\right) r\left(B^{\prime}\right)\left(t_{B^{\prime}, A^{\prime}}+t_{A^{\prime}, B^{\prime}}^{0}\right) \\
=\operatorname{deg}\left(A^{\prime}\right) r\left(B^{\prime}\right)-\operatorname{deg}\left(B^{\prime}\right) r\left(A^{\prime}\right)+r\left(A^{\prime}\right) r\left(B^{\prime}\right)\left(t_{A^{\prime}, B^{\prime}}-p_{0}\right),
\end{gathered}
$$

by Lemma $2.4(4)$. Hence, $\mu_{H_{\text {om }}\left(B^{\prime}{ }_{*}, A^{\prime}{ }_{*}\right)}=\mu_{A}^{\prime}-\mu_{B}^{\prime}+t_{A^{\prime}, B^{\prime}}-p_{0}$ and therefore

$$
\mu_{H_{\text {om }}\left(B^{\prime}{ }_{*}, A^{\prime} * \otimes K(P)\right)}=\mu_{A}^{\prime}-\mu_{B}^{\prime}+t_{A^{\prime}, B^{\prime}}+2 g-2 .
$$

Thus $\mu_{H_{o m} m_{s t}\left(B^{\prime}, A^{\prime}{ }_{*} \otimes K(P)\right)} \geq g-1$ (respectively, $\mu_{H o m_{s t}\left(B^{\prime}{ }_{*}, A^{\prime}{ }_{*} \otimes K(P)\right)} \leq g-1$ ) if $\mu_{B}^{\prime}-\mu_{A}^{\prime} \leq g-1+t_{A^{\prime}, B^{\prime}}$ (respectively, if $\left.\mu_{B}^{\prime}-\mu_{A}^{\prime} \geq g-1+t_{A^{\prime}, B^{\prime}}\right)$. By Serre duality, $H^{0}\left(\operatorname{Hom}\left(A^{\prime}{ }_{*}, B^{\prime}{ }_{*}\right)\right)=0$ if and only if $H^{1}\left(\operatorname{Hom}_{s t}\left(B^{\prime}{ }_{*}, A^{\prime}{ }_{*} \otimes K(P)\right)\right)=0$. Hence (A)(1) may be restated as

$$
H^{1}\left(\operatorname{Hom}_{s t}\left(B^{\prime}{ }_{*}, A^{\prime}{ }_{*} \otimes K(P)\right)\right)=0
$$

if $\mu_{H_{o m} m_{s t}\left(B^{\prime}{ }_{*}, A^{\prime} * \otimes K(P)\right)} \geq g-1$. Since $A_{*}^{\prime} \otimes K(P)$ is a general bundle if $A_{*}^{\prime}$ is so, taking $A_{*}=B^{\prime}{ }_{*}$ and $B_{*}=A^{\prime}{ }_{*} \otimes K(P)$ we get $H^{1}\left(\operatorname{Hom}_{s t}\left(A_{*}, B_{*}\right)\right)=0$ if $\mu_{H o m_{s t}}\left(A_{*}, B_{*}\right) \geq$ $g-1$ (that is, $\mu_{B}-\mu_{A} \geq g-1+t_{A, B}+t_{A, B}^{0}$ ) for general bundles $A_{*}, B_{*}$.

Similarly $(\mathrm{B})(1)$ can be deduced from (A)(2). It only remains to prove (A)(2).

(A)(2). Let $\mu_{B}-\mu_{A} \geq g-1+t_{A, B}$. Since $t_{A, B} \geq 0$, one has

$$
H^{1}(\operatorname{Hom}(A, B))=0
$$

([Hi, Theorem 4.6]). Also $\chi\left(\operatorname{Hom}\left(A_{*}, B_{*}\right)\right) \geq 0$ in this case, which means that

$$
H^{0}\left(\operatorname{Hom}\left(A_{*}, B_{*}\right)\right) \neq 0
$$


provided $H^{1}\left(\operatorname{Hom}\left(A_{*}, B_{*}\right)\right) \neq 0$. Let $f: A_{*} \rightarrow B_{*}$ be a nonzero parabolic homomorphism and $h: B_{*} \rightarrow A_{*} \otimes K(P)$ be a strongly parabolic homomorphism. As in (A)(1), we conclude that $(\mathrm{A})(2)$ holds for $r(B)=1$. Assume that $r(B) \geq 2$.

Case 2 (i) $\mu_{B}-\mu_{A} \geq g+t_{A, B}$.

We choose $B_{1}$ and $B_{2}$ with ranks and weights as in the proof of (A)(1). Choose $d_{1}$ satisfying

$$
\operatorname{deg}(B)-r_{2}\left(\mu_{A}+g-1+t_{A, B_{2}}\right) \geq d_{1} \geq r_{1}\left(\mu_{A}+g-1+t_{A, B_{1}}\right) .
$$

Then $\mu_{B_{2}}-\mu_{A} \geq g-1+t_{A, B_{2}}$ and $\mu_{B_{1}}-\mu_{A} \geq g-1+t_{A, B_{1}}$. The result can now be proved using induction and semicontinuity as done in case 1(i).

Case 2 (ii) $g-1+t_{A, B} \leq \mu_{B}-\mu_{A}<g+t_{A, B}$.

We claim that any nonzero parabolic homomorphism $f: A_{*} \longrightarrow B_{*}$ is a generic surjection. Indeed, if it is not, then by elementary transformation of $B_{*}$ outside parabolic points, we can find a general bundle $B_{*}^{\prime}$ such that the image of $f$ is contained in $B_{*}^{\prime}$, with $r(B)=r\left(B^{\prime}\right)$ and $\operatorname{deg}\left(B^{\prime}\right) \leq r(B)\left(g-1+t_{A, B}+\mu_{A}\right)$. Then by $(\mathrm{A})(1)$, we have

$$
H^{0}\left(\operatorname{Hom}\left(A_{*}, B^{\prime}{ }_{*}\right)\right)=0
$$

so that $f=0$. We therefore conclude that any nonzero $f$ is a generic surjection.

For $h: B_{*} \longrightarrow A_{*} \otimes K(P)$, by Proposition 3.3,

$$
h \circ f=0 .
$$

Hence the generic surjectivity of $f$ implies that $h=0$, which is a contradiction. This completes the proof of the theorem.

Proposition 4.3. Let $E_{*}^{\prime}$ and $E_{*}^{\prime \prime}$ be general stable parabolic vector bundles of slopes $\mu^{\prime}$ and $\mu^{\prime \prime}$, and ranks $r^{\prime}$ and $r^{\prime \prime}$ with $r^{\prime}+r^{\prime \prime}=r$ and $\mu^{\prime \prime}-\mu^{\prime} \geq g-1+t_{E^{\prime}, E^{\prime \prime}}$ and with the fixed parabolic structures. Then a general parabolic extension $E_{*}$ of $E_{*}^{\prime \prime}$ by $E_{*}^{\prime}$ is a (general) parabolic stable vector bundle.

Proof. In a neighborhood of $F_{*}=E_{*}^{\prime} \oplus E_{*}^{\prime \prime}$, the extensions of a deformation of $E_{*}^{\prime \prime}$ by a deformation of $E_{*}^{\prime}$ occur in a family parametrized by a smooth germ $W$ whose tangent space has the following description

$$
T_{F} W=H^{1}\left(H o m\left(E_{*}^{\prime}, E_{*}^{\prime}\right)\right) \oplus H^{1}\left(H o m\left(E_{*}^{\prime \prime}, E_{*}^{\prime \prime}\right)\right) \oplus H^{1}\left(H o m\left(E_{*}^{\prime \prime}, E_{*}^{\prime}\right)\right) .
$$

One has

$$
H^{1}\left(\operatorname{Hom}\left(E_{*}, E_{*}\right)\right)
$$

$\cong H^{1}\left(\operatorname{Hom}\left(E_{*}^{\prime}, E_{*}^{\prime}\right)\right) \oplus H^{1}\left(\operatorname{Hom}\left(E_{*}^{\prime \prime}, E_{*}^{\prime \prime}\right)\right) \oplus H^{1}\left(\operatorname{Hom}\left(E_{*}^{\prime \prime}, E_{*}^{\prime}\right)\right) \oplus H^{1}\left(H o m\left(E_{*}^{\prime}, E_{*}^{\prime \prime}\right)\right)$.

By Theorem 4.2, $H^{1}\left(\operatorname{Hom}\left(E_{*}^{\prime}, E_{*}^{\prime \prime}\right)\right)=0$ as $\mu^{\prime \prime}-\mu^{\prime} \geq g-1+t_{E^{\prime}, E^{\prime \prime}}$. It follows that the map $T_{F}(W) \rightarrow H^{1}\left(\operatorname{Hom}\left(E_{*}, E_{*}\right)\right)$ is an isomorphism, so that the family parametrized by $W$ is a versal family. Every parabolic vector bundle can be deformed to a stable parabolic vector bundle. Therefore, using openness of versality it follows that a general bundle in this family is a parabolic stable bundle. 
5. The stratification of $M(r, d)$. Let $E_{*}$ be a parabolic vector bundle of rank $r$ and degree $d$ with rational parabolic weights $\alpha_{i}(p)$ of multiplicities $n_{i}(p)$ for $p \in P$. Let $w=w t E$. Fix integers $r^{\prime}, r^{\prime \prime}=r-r^{\prime}$ and $\left\{n_{i}^{\prime}(p)\right\}_{p \in P}$ with $1 \leq r^{\prime}<r$,

$$
0 \leq n_{i}^{\prime}(p) \leq n_{i}(p)
$$

and $\sum_{i} n_{i}^{\prime}(p)=r^{\prime}$ for all $p \in P$. Let $E_{*}^{\prime}$ denote a parabolic subbundle of $E_{*}$ of rank $r^{\prime}$, degree $d^{\prime}$ with $n_{i}^{\prime}(p)$ the multiplicity of $\alpha_{i}(p), p \in P$, equipped with the induced parabolic structure. Recall that we take $n_{i}^{\prime}(p)=0$ if $\alpha_{i}(p)$ is not a weight of $E_{*}^{\prime}$. Note that for all such subbundles, $w^{\prime}=w t\left(E^{\prime}\right)$ and $t_{n^{\prime}, n^{\prime \prime}}=t_{E^{\prime}, E / E^{\prime}}$ are kept fixed. Let $s^{p a r}\left(E_{*}, E_{*}^{\prime}\right)=r^{\prime} d-r d^{\prime}$.

DEFINITION 5.1. If $E_{*}$ has a subbundle $E_{*}^{\prime}$ of rank $r^{\prime}$ and induced parabolic structure with weights $\alpha_{i}(p)$ of multiplicity $n_{i}^{\prime}(p), p \in P$, define

$$
s_{r^{\prime}}^{\text {par }}\left(E_{*}\right)=\min s^{\text {par }}\left(E_{*}, E_{*}^{\prime}\right),
$$

where the minimum is taken over all parabolic subbundles $E_{*}^{\prime} \subset E_{*}$ of rank $r^{\prime}$ and weights $\alpha_{i}(p)$ of multiplicity $n_{i}^{\prime}(p), p \in P$.

One has $s_{r^{\prime}}^{p a r}\left(E_{*}\right) \equiv r^{\prime} d \bmod r$. There is an upper bound on $s_{r^{\prime}}^{p a r}\left(E_{*}\right)$ given by

$$
s_{r^{\prime}}^{p a r}\left(E_{*}\right) \leq r^{\prime} r^{\prime \prime}\left(g+t_{n^{\prime}, n^{\prime \prime}}\right)
$$

(Proposition 3.7(1)). If $E_{*}$ is stable, then $s_{r^{\prime}}^{p a r}\left(E_{*}\right)>r w^{\prime}-r^{\prime} w$. By Proposition 4.1(1), for a general parabolic bundle $E_{*}$, one has $s_{r^{\prime}}^{\text {par }}\left(E_{*}\right) \geq r^{\prime}\left(r-r^{\prime}\right)\left((g-1)+t_{n^{\prime}, n^{\prime \prime}}\right)$. Let $s_{\text {max }}^{\text {par }}, s_{\text {min }}^{\text {par }}$ denote the maximum and minimum of possible values of $s_{r^{\prime}}^{\text {par }}$.

5.1. The subsets $W_{r^{\prime}, s}^{\text {par }}, V_{r^{\prime}, s}^{\text {par }}$ and $U_{r^{\prime}, s}^{\text {par }}$. Fix an integer $s$ such that $s_{\text {min }}^{\text {par }} \leq$ $s \leq s_{\text {max }}^{\text {par }}, s \equiv r^{\prime} d \bmod r$. Let $d^{\prime}=\left(r^{\prime} d-s\right) / r, d^{\prime \prime}=d-d^{\prime}, \mu^{\prime}=d^{\prime} / r^{\prime}, \mu^{\prime \prime}=d^{\prime \prime} / r^{\prime \prime}$ and $\mu=d / r$. In the following we always assume that $E_{*}^{\prime}$ is of rank $r^{\prime}$, degree $d^{\prime}$ and parabolic weights $\alpha_{i}(p)$ with multiplicities $n_{i}^{\prime}(p), p \in P$, and $E_{*}^{\prime \prime}$ has rank $r^{\prime \prime}$, degree $d^{\prime \prime}$ and weights $\alpha_{i}(p)$ with multiplicities $n_{i}^{\prime \prime}(p)=n_{i}(p)-n_{i}^{\prime}(p)$.

Let $M_{r^{\prime}, s}$ be the subset of $M(r, d)$ defined by

$$
M_{r^{\prime}, s}=\left\{E_{*} \in M(r, d) \mid s_{r^{\prime}}^{\text {par }}\left(E_{*}\right)=s\right\} .
$$

Let $W_{r^{\prime}, s}^{\text {par }}$ denote the subset of $M(r, d)$ consisting of stable parabolic bundles $E_{*}$ which are extensions of a stable parabolic vector bundle $E_{*}^{\prime \prime}$ by a stable parabolic vector bundle $E_{*}^{\prime}$. The construction of $W_{r^{\prime}, s}^{p a r}$ in the parabolic case works similarly as that of the corraesponding set $W_{r^{\prime}, s}$ in the case of ordinary vector bundles ([La, Section 4], [RT, 1.3], [BL]); one has to replace $H^{1}\left(\operatorname{Hom}\left(E^{\prime \prime}, E^{\prime}\right)\right)$ by $H^{1}\left(H o m\left(E_{*}^{\prime \prime}, E_{*}^{\prime}\right)\right)$ to extend the construction to the case of parabolic vector bundles. As $M\left(r^{\prime}, d^{\prime}\right)$ and $M\left(r^{\prime \prime}, d^{\prime \prime}\right)$ are irreducible and smooth, $W_{r^{\prime}, s}^{p a r}$ is irreducible. By Proposition 4.3, $W_{r^{\prime}, s}^{p a r}$ is nonempty for $s \geq r^{\prime} r^{\prime \prime}\left((g-1)+t_{n^{\prime}, n^{\prime \prime}}\right)$.

Let $V_{r^{\prime}, s}^{p a r}$ be the subset of $M(r, d)$ consisting of stable parabolic bundles which are extensions of parabolic vector bundles $E_{*}^{\prime \prime}$ by parabolic vector bundles $E_{*}^{\prime}$ (the parabolic bundles $E_{*}^{\prime}, E_{*}^{\prime \prime}$ are not necessarily stable). One has $W_{r^{\prime}, s}^{p a r} \subset V_{r^{\prime}, s}^{p a r}$ and $M_{r^{\prime}, s} \subset V_{r^{\prime}, s}^{p a r}$.

For a fixed $E_{*}$, let $A_{r^{\prime}, d^{\prime}}^{p a r}\left(E_{*}\right)=\left\{E_{*}^{\prime} \mid E_{*}^{\prime} \subset E_{*}\right.$ parabolic subbundle of rank $r^{\prime}$, degree $d^{\prime}$ and weight multiplicities $\left.n_{i}^{\prime}(p), p \in P\right\}$.

Lemma 5.2. Assume that $W_{r^{\prime}, s}^{\text {par }}$ is nonempty. Let $E_{*}$ be a general element in $W_{r^{\prime}, s}^{\text {par }}$. 
(1) If $s \leq r^{\prime} r^{\prime \prime}\left((g-1)+t_{n^{\prime}, n^{\prime \prime}}\right)$, then $E_{*}$ has only finitely many subbundles of rank $r^{\prime}$, degree $d^{\prime}$ and of given parabolic structure, i.e., $\operatorname{dim} A_{r^{\prime}, d^{\prime}}^{\text {par }}\left(E_{*}\right)=0$, and

$$
\operatorname{dim} W_{r^{\prime}, s}^{\text {par }}=\operatorname{dim} M(r, d)+s-r^{\prime} r^{\prime \prime}\left((g-1)+t_{n^{\prime}, n^{\prime \prime}}\right) .
$$

(2) If $s \geq r^{\prime} r^{\prime \prime}\left((g-1)+t_{n^{\prime}, n^{\prime \prime}}\right)$, then

$$
\begin{gathered}
\operatorname{dim} A_{r^{\prime}, d^{\prime}}^{\text {par }}\left(E_{*}\right)=s-r^{\prime} r^{\prime \prime}\left((g-1)+t_{n^{\prime}, n^{\prime \prime}}\right), \\
\operatorname{dim} W_{r^{\prime}, s}^{p a r}=r^{2}(g-1)+1+r^{2} t_{n, n}=\operatorname{dim} M(r, d) .
\end{gathered}
$$

Proof. By the construction of $W_{r^{\prime}, s}^{\text {par }}$, there is a morphism $p$ from an irreducible variety $\mathbf{P}$ onto $W_{r^{\prime}, s}^{\text {par }}$ with

$$
\begin{gathered}
\operatorname{dim} \mathbf{P}=\operatorname{dim} M\left(r^{\prime}, d^{\prime}\right)+\operatorname{dim} M\left(r^{\prime \prime}, d^{\prime \prime}\right)+h^{1}\left(\operatorname{Hom}\left(E_{*}^{\prime \prime}, E_{*}^{\prime}\right)\right)-1 \\
=r^{\prime 2}(g-1)+1+{r^{\prime}}^{2} t_{n^{\prime}, n^{\prime}}+r^{\prime \prime 2}(g-1)+r^{\prime \prime 2} t_{n^{\prime \prime}, n^{\prime \prime}}+1+r^{\prime} r^{\prime \prime}\left(\mu^{\prime \prime}-\mu^{\prime}+g-1+t_{n^{\prime \prime}, n^{\prime}}\right)-1 \\
=r^{2}(g-1)+1+s-r^{\prime} r^{\prime \prime}(g-1)+r^{\prime 2} t_{n^{\prime}, n^{\prime}}+r^{\prime \prime 2} t_{n^{\prime \prime}, n^{\prime \prime}}+r^{\prime} r^{\prime \prime} t_{n^{\prime \prime}, n^{\prime}} \\
=\operatorname{dim} M(r, d)+s-r^{\prime} r^{\prime \prime}\left((g-1)+t_{n^{\prime}, n^{\prime \prime}}\right) .
\end{gathered}
$$

The last step is obtained by using Lemma 2.4(1).

A general parabolic bundle $E_{*}$ of $W_{r^{\prime}, s}^{p a r}$ is an extension of $E_{*}^{\prime \prime}$ by $E_{*}^{\prime}$, with $E_{*}^{\prime}$ and $E_{*}^{\prime \prime}$ being general stable parabolic vector bundles. The fiber of $p$ at $E_{*}$ is $A_{r^{\prime}, d^{\prime}}^{p a r}\left(E_{*}\right)$. The set $A_{r^{\prime}, d^{\prime}}^{\text {par }}\left(E_{*}\right)$ can be identified with the parabolic Quot scheme of $E_{*}$ corresponding to torsionfree quotients $E_{*}^{\prime \prime}$ of $E_{*}$ of rank $r^{\prime \prime}$, degree $d^{\prime \prime}$ and fixed parabolic structure. The tangent space to this Quot scheme at $E_{*}^{\prime \prime}$ is $H^{0}\left(H o m\left(E_{*}^{\prime}, E_{*}^{\prime \prime}\right)\right)$.

(1) For general bundles $E_{*}^{\prime}$ and $E_{*}^{\prime \prime}$, one has

$$
H^{0}\left(\operatorname{Hom}\left(E_{*}^{\prime}, E_{*}^{\prime \prime}\right)\right)=0
$$

for $s \leq r^{\prime} r^{\prime \prime}\left((g-1)+t_{n^{\prime}, n^{\prime \prime}}\right)$ (Theorem 4.2). Hence $\operatorname{dim} A_{r^{\prime}, d^{\prime}}^{p a r}=0$ and thus $A_{r^{\prime}, d^{\prime}}^{p a r}$ has finitely many points.

Hence $\operatorname{dim} W_{r^{\prime}, d^{\prime}}^{\text {par }}=\operatorname{dim} \mathbf{P}$, and the result follows.

(2) For $s \geq r^{\prime} r^{\prime \prime}\left((g-1)+t_{n^{\prime}, n^{\prime \prime}}\right)$, one has

$$
\operatorname{dim} H^{1}\left(\operatorname{Hom}\left(E_{*}^{\prime}, E_{*}^{\prime \prime}\right)\right)=0
$$

(Theorem 4.2), so that $h^{0}\left(\operatorname{Hom}\left(E_{*}^{\prime}, E_{*}^{\prime \prime}\right)\right)=s-r^{\prime} r^{\prime \prime}\left((g-1)+t_{n^{\prime}, n^{\prime \prime}}\right)$. Hence for $s \geq r^{\prime} r^{\prime \prime}\left(g-1+t_{n^{\prime}, n^{\prime \prime}}\right)$,

$$
\operatorname{dim} A_{r^{\prime}, d^{\prime}}^{p a r}\left(E_{*}\right)=s-r^{\prime} r^{\prime \prime}\left((g-1)+t_{n^{\prime}, n^{\prime \prime}}\right)
$$

at general points. Thus the general fibers of $p$ have dimension equal to $s-r^{\prime} r^{\prime \prime}((g-$ 1) $\left.+t^{n^{\prime}, n^{\prime \prime}}\right)$ and

$$
\operatorname{dim} W_{r^{\prime}, s}^{\text {par }}=\operatorname{dim} \mathbf{P}-\operatorname{dim} A_{r^{\prime}, s}^{\text {par }}\left(E_{*}\right)=\operatorname{dim} M(r, d) .
$$


LEMMA 5.3.

1. If $V_{r^{\prime}, s}^{\text {par }}$ is nonempty, then $W_{r^{\prime}, s}^{\text {par }}$ is nonempty.

2. $V_{r^{\prime}, s}^{\text {par }}$ is contained in the closure of $W_{r^{\prime}, s}^{\text {par }}$.

3. $\operatorname{dim} V_{r^{\prime}, s}^{p a r}=\operatorname{dim} W_{r^{\prime}, s}^{p a r}$, and $V_{r^{\prime}, s}^{p a r}$ is irreducible.

Proof. The proof is similar to that of the corresponding result for ordinary vector bundles ([Tb], [RT, Proposition 1.6]). One shows that any parabolic bundle $E_{0 *} \in V_{r^{\prime}, s}^{p a r}$ can be deformed to an element of $W_{r^{\prime}, s}^{p a r}$. Suppose that $E_{0 *}$ is an extension of $E_{*}^{\prime \prime}$ by $E_{*}^{\prime}$. There exist irreducible families $T^{\prime}$ and $T^{\prime \prime}$ of parabolic vector bundles containing $E_{*}^{\prime}$ and $E_{*}^{\prime \prime}$ respectively such that the generic members are stable parabolic vector bundles. Let $T$ be the family of extensions of bundles in $T^{\prime \prime}$ by bundles in $T^{\prime}$ and $T^{s}$ its subset giving stable parabolic bundles. Since $E_{0 *} \in T^{s}$, the general member of $T^{s}$ is in $W_{r^{\prime}, s}^{\text {par }}$.

LEMMA 5.4.

1. Let

$$
0 \longrightarrow E_{*}^{\prime} \longrightarrow E_{*} \longrightarrow E_{*}^{\prime \prime} \longrightarrow 0
$$

be an exact sequence of parabolic vector bundles with $E_{*}$ stable. Then, there exists an exact sequence of parabolic vector bundles

$$
0 \longrightarrow E_{1 *}^{\prime} \longrightarrow E_{1 *} \longrightarrow E_{1 *}^{\prime \prime} \longrightarrow 0
$$

such that $E_{1 *}$ is stable, has same rank, degree and parabolic structure as $E_{*}$, also $\operatorname{deg}\left(E_{1}^{\prime}\right)=\operatorname{deg}\left(E^{\prime}\right)-1, \operatorname{deg}\left(E_{1}^{\prime \prime}\right)=\operatorname{deg}\left(E^{\prime \prime}\right)+1$ and $E_{1 *}^{\prime}$ (respectively, $\left.E_{1 *}^{\prime \prime}\right)$ has the same parabolic structures as $E_{*}^{\prime}$ (respectively, $\left.E_{*}^{\prime \prime}\right)$.

2. If $V_{r, s}^{p a r}$ is nonempty for some $s=s_{1}$, then it is nonempty for all possible values $s \geq s_{1}$.

Proof. (1) This can be proved by using elementary transformations and dual elementary transformations at non-parabolic points, see [RT, Proposition 1.11] for details.

(2) This can be proved by using (1) repeatedly $s-s_{1}$ times.

Proposition 5.5. Suppose that $V_{r^{\prime}, s}^{\text {par }}$ is nonempty. Then

1. $M_{r^{\prime}, s}$ is nonempty for $s \leq r^{\prime} r^{\prime \prime}\left(\stackrel{g}{g}-1+t_{n^{\prime}, n^{\prime \prime}}\right)+r-1$;

2. $\operatorname{dim} M_{r^{\prime}, s}=r^{2}(g-1)+1+r^{2} t_{n, n}+s-r^{\prime} r^{\prime \prime}\left(g-1+t_{n^{\prime}, n^{\prime \prime}}\right)$.

Proof. Any $E_{*} \in V_{r^{\prime}, s}^{p a r}$ is an extension of $E_{*}^{\prime \prime}$ by $E_{*}^{\prime}$, and hence $E_{*}$ can have a maximum subbundle $E_{0 *}^{\prime}$ with degree $\left(E_{0 *}^{\prime}\right) \geq \operatorname{degree}\left(E^{\prime}\right)$, so that $E_{*} \in M_{r^{\prime}, s^{\prime}}, s^{\prime} \leq s$. Thus,

$$
V_{r^{\prime}, s}^{p a r} \subset \coprod_{s^{\prime} \leq s} M_{r^{\prime}, s^{\prime}}
$$

and hence

$$
V_{r^{\prime}, s}^{p a r}=M_{r^{\prime}, s} \coprod\left(\coprod_{s^{\prime}<s}\left(M_{r^{\prime}, s^{\prime}} \cap V_{r^{\prime}, s}^{p a r}\right)\right) .
$$

Since $M_{r^{\prime}, s^{\prime}} \subset V_{r^{\prime}, s^{\prime}}^{p a r}$, we have $\operatorname{dim} M_{r^{\prime}, s^{\prime}} \leq \operatorname{dim} V_{r^{\prime}, s^{\prime}}^{p a r} \forall s^{\prime}$. Hence

$$
\operatorname{dim} M_{r^{\prime}, s^{\prime}} \cap V_{r^{\prime}, s}^{\text {par }} \leq \operatorname{dim} M_{r^{\prime}, s^{\prime}} \leq \operatorname{dim} V_{r^{\prime}, s^{\prime}}^{\text {par }} .
$$


By Lemma 5.2 and Lemma 5.3,

$$
\operatorname{dim} V_{r^{\prime}, s^{\prime}}^{\text {par }}<\operatorname{dim} V_{r^{\prime}, s}^{\text {par }}
$$

for $s^{\prime}<s$. It follows that $\operatorname{dim} \coprod_{s^{\prime}<s}\left(M_{r^{\prime}, s^{\prime}} \cap V_{r^{\prime}, s}^{\text {par }}\right)<\operatorname{dim} V_{r^{\prime}, s}^{\text {par }}$. Then Eqn. (5.1) implies that $M_{r^{\prime}, s}$ is nonempty and a general element of $V_{r^{\prime}, s}^{p a r}$ belongs to $M_{r^{\prime}, s}$. Hence $\operatorname{dim} M_{r^{\prime}, s}=\operatorname{dim} V_{r^{\prime}, s}^{p a r}$. The second part now follows from Lemma 5.2 and Lemma 5.3.

\subsection{Nonemptiness of the strata.}

Proposition 5.6. Let $E_{*}^{\prime}$ and $E_{*}^{\prime \prime}$ be general stable parabolic vector bundles of rank $r^{\prime}, r^{\prime \prime}$, degree $d^{\prime}, d^{\prime \prime}$ respectively, with $r=r^{\prime}+r^{\prime \prime}, d=d^{\prime}+d^{\prime \prime}, w t E^{\prime}=w^{\prime}$, and $w t E^{\prime \prime}=w^{\prime \prime}$. Let

$$
r^{\prime \prime} w^{\prime}-r^{\prime} w^{\prime \prime}<r^{\prime} d-r d^{\prime} \leq r+r^{\prime \prime} w^{\prime}-r^{\prime} w^{\prime \prime}
$$

Then

(A) A parabolic vector bundle $E_{*}$ which is a general extension of $E_{*}^{\prime \prime}$ by $E_{*}^{\prime}$ is stable for $g \geq 1+\left(r^{\prime \prime} p_{0}-\left[w^{\prime \prime}\right]\right) / r^{\prime}$, where $p_{0}=\# P$ is the number of parabolic points and $\left[w^{\prime \prime}\right]$ denotes the integral part of $w^{\prime \prime}$. One has $s_{0}:=s_{r^{\prime}}^{\text {par }}\left(E_{*}\right)=s_{\text {min }}^{\text {par }}$.

(B) If $r^{\prime \prime}=1$, then (A) holds for $g \geq 2$.

Proof. Note that Eqn. (5.2) is equivalent to

$$
\begin{aligned}
& \mu\left(E_{*}\right)<\mu\left(E_{*}^{\prime \prime}\right) \leq \mu\left(E_{*}\right)+\frac{1}{r^{\prime \prime}} \\
& \mu\left(E_{*}^{\prime}\right)<\mu\left(E_{*}\right) \leq \mu\left(E_{*}^{\prime}\right)+\frac{1}{r^{\prime}} .
\end{aligned}
$$

Suppose that $E_{*}$ is not stable. Then it has a destabilizing subsheaf $F_{*}$ with $\mu\left(F_{*}\right) \geq \mu\left(E_{*}\right)$. Choose $F_{*}$ having maximum parabolic slope. Consider the composite homomorphism

$$
h: F_{*} \hookrightarrow E_{*} \longrightarrow E_{*}^{\prime \prime} .
$$

If $h=0$, then $F_{*} \subset E_{*}^{\prime}, \mu\left(F_{*}\right) \leq \mu\left(E_{*}^{\prime}\right)$ as $E_{*}^{\prime}$ is stable. But $\mu\left(F_{*}\right) \geq \mu\left(E_{*}\right)$ and $\mu\left(E_{*}\right)>\mu\left(E_{*}^{\prime}\right)$ by Eqn. (5.4) so that $\mu\left(F_{*}\right)>\mu\left(E_{*}^{\prime}\right)$, a contradiction. Hence $h \neq 0$. Let $F_{*}^{\prime \prime}$ and $F_{*}^{\prime}$ respectively be the image and kernel of $h$.

Claim 1: $r\left(F^{\prime \prime}\right)=r^{\prime \prime}$.

For proving this, one may assume that $F_{*}$ is stable by replacing $F_{*}$ with a subbundle of the same parabolic slope and minimum rank. Assume that $r\left(F^{\prime \prime}\right)<r^{\prime \prime}$. Since $E_{*}^{\prime \prime}$ is a general bundle, by Proposition 4.1 one has

$$
\mu_{E^{\prime \prime}}-\mu_{F^{\prime \prime}} \geq\left(1-\frac{r\left(F^{\prime \prime}\right)}{r^{\prime \prime}}\right)\left(g-1+t_{F^{\prime \prime}, E^{\prime \prime} / F^{\prime \prime}}\right) .
$$

This is equivalent to

$$
\mu\left(E_{*}^{\prime \prime}\right) \geq \mu\left(F_{*}^{\prime \prime}\right)+\frac{w^{\prime \prime}}{r^{\prime \prime}}-\frac{w t F^{\prime \prime}}{r\left(F^{\prime \prime}\right)}+\frac{r^{\prime \prime}-r\left(F^{\prime \prime}\right)}{r^{\prime \prime}}\left(g-1+t_{F^{\prime \prime}, E^{\prime \prime} / F^{\prime \prime}}\right) .
$$


By Eqn. (5.3), one has $\mu\left(E_{*}^{\prime \prime}\right) \leq \mu\left(E_{*}\right)+\frac{1}{r^{\prime \prime}} \leq \mu\left(F_{*}\right)+\frac{1}{r^{\prime \prime}}$. Since $F_{*}$ is stable, $\mu\left(F_{*}\right) \leq \mu\left(F_{*}^{\prime \prime}\right)$. Thus

$$
\mu\left(F_{*}^{\prime \prime}\right)+\frac{1}{r^{\prime \prime}} \geq \mu\left(E_{*}^{\prime \prime}\right),
$$

and the equality holds only if $F_{*}=F_{*}^{\prime \prime}$. Now, Eqn. (5.6) and Eqn. (5.7) give

$$
\mu\left(F_{*}^{\prime \prime}\right)+\frac{1}{r^{\prime \prime}} \geq \mu\left(E_{*}^{\prime \prime}\right) \geq \mu\left(F_{*}^{\prime \prime}\right)+\frac{1}{r^{\prime \prime}}+T_{1}+T_{2},
$$

where $T_{1}=\left(w^{\prime \prime}\right) / r^{\prime \prime}-\left(w t F^{\prime \prime}\right) / r\left(F^{\prime \prime}\right)+t_{F^{\prime \prime}, E^{\prime \prime} / F^{\prime \prime}}\left(r^{\prime \prime}-r\left(F^{\prime \prime}\right)\right) / r^{\prime \prime}$ and

$$
T_{2}=\frac{(g-2)\left(r^{\prime \prime}-r\left(F^{\prime \prime}\right)\right)}{r^{\prime \prime}}+\frac{r^{\prime \prime}-r\left(F^{\prime \prime}\right)-1}{r^{\prime \prime}} .
$$

Note that

$r^{\prime \prime} r\left(F^{\prime \prime}\right) T_{1}=r\left(F^{\prime \prime}\right) w t\left(E^{\prime \prime} / F^{\prime \prime}\right)-\left(r^{\prime \prime}-r\left(F^{\prime \prime}\right)\right) w t F^{\prime \prime}+\left(r^{\prime \prime}-r\left(F^{\prime \prime}\right)\right) r\left(F^{\prime \prime}\right) t_{F^{\prime \prime}, E^{\prime \prime} / F^{\prime \prime}}$.

Hence $T_{1}$ is nonnegative and it is zero if and only if at each $p \in P$ both $E_{*}^{\prime \prime}$ and $F_{*}^{\prime \prime}$ have same unique parabolic weight with multiplicity $r^{\prime \prime}$ and $r\left(F^{\prime \prime}\right)$ respectively (Lemma 2.5(2)). In particular, for $T_{1}=0$, we have

$$
\frac{w t E^{\prime \prime}}{r^{\prime \prime}}=\frac{w t F^{\prime \prime}}{r\left(F^{\prime \prime}\right)}
$$

and $t_{F^{\prime \prime}, E^{\prime \prime} / F^{\prime \prime}}=0$. For $g \geq 2$, one has $T_{2} \geq 0$, with the equality being valid only if $g=2$ as well as $r\left(F^{\prime \prime}\right)=r^{\prime \prime}-1$. Clearly, if $T_{1}+T_{2}>0$, then Eqn. (5.8) gives a contradiction.

Suppose that $T_{1}=T_{2}=0$. Then all the above inequalities are equalities so that

$$
\mu\left(E_{*}^{\prime \prime}\right)=\mu\left(F_{*}^{\prime \prime}\right)+\frac{1}{r^{\prime \prime}}=\mu\left(F_{*}\right)+\frac{1}{r^{\prime \prime}}=\mu\left(E_{*}\right)+\frac{1}{r^{\prime \prime}} .
$$

Thus, $\mu\left(F_{*}^{\prime \prime}\right)=\mu\left(F_{*}\right)$ which, by the stability condition of $F_{*}$, implies that $F_{*} \cong F_{*}^{\prime \prime}$ and $F_{*}^{\prime}=0$. One has $\mu_{E^{\prime \prime}}-\mu_{F^{\prime \prime}}=\mu\left(E_{*}^{\prime \prime}\right)-\mu\left(F_{*}^{\prime \prime}\right)=1 / r^{\prime \prime}$. It follows that $F_{*}^{\prime \prime}$ is a maximal subbundle of $E_{*}^{\prime \prime}$ (of given fixed parabolic type) and $s_{r^{\prime \prime}-1}^{p}\left(E_{*}^{\prime \prime}\right)=r^{\prime \prime}-1$. Since $E_{*}^{\prime \prime}$ is a general stable bundle, by Proposition 4.1 there are finitely many $F_{*}^{\prime \prime}=F_{*}$ possible, say $F_{1 *}, \cdots, F_{m *}$, and they are general parabolic bundles.

Let

$$
[e]:=\left[E_{*}\right] \in H^{1}\left(\operatorname{Hom}\left(E_{*}^{\prime \prime}, E_{*}^{\prime}\right)\right)
$$

be the extension class for the exact sequence

$$
0 \longrightarrow E_{*}^{\prime} \longrightarrow E_{*} \longrightarrow E_{*}^{\prime \prime} \longrightarrow 0 .
$$

Consider $F_{*}=F_{i *}$. Since $h_{i}: F_{*} \rightarrow E_{*}^{\prime \prime}$ lifts to $F_{*} \rightarrow E_{*}$, the cohomology class $[e]$ defined in Eqn. (5.9) is in the kernel of the map

$$
\bar{h}_{i}: H^{1}\left(\operatorname{Hom}\left(E_{*}^{\prime \prime}, E_{*}^{\prime}\right)\right) \longrightarrow H^{1}\left(\operatorname{Hom}\left(F_{*}, E_{*}^{\prime}\right)\right) .
$$

Since $\mu\left(E_{*}\right)=\mu\left(F_{*}\right)>\mu\left(E_{*}^{\prime}\right)$ (by Eqn. (5.4)), and $F_{*}, E_{*}^{\prime}$ are stable, we have

$$
H^{0}\left(\operatorname{Hom}\left(F_{*}, E_{*}^{\prime}\right)\right)=0 .
$$


Then

$$
\begin{gathered}
h^{1}\left(\operatorname{Hom}\left(F_{*}, E_{*}^{\prime}\right)\right)=r^{\prime} d(F)-r\left(F_{*}\right) d^{\prime}+r^{\prime}\left(r^{\prime \prime}-1\right)+\left(t_{F, E^{\prime}}\right) r^{\prime}\left(r^{\prime \prime}-1\right) \\
=r^{\prime}\left(r^{\prime \prime}-1\right)\left(\mu(F)-\mu\left(E^{\prime}\right)+1+t_{F, E^{\prime}}\right) .
\end{gathered}
$$

Since $\mu\left(F_{*}\right)>\mu\left(E_{*}^{\prime}\right)$, we have $\mu(F)-\mu\left(E^{\prime}\right)>\frac{w t E^{\prime}}{r^{\prime}}-\frac{w t F}{r^{\prime \prime}-1}$. Hence

$$
h^{1}\left(\operatorname{Hom}\left(F_{*}, E_{*}^{\prime}\right)\right)>r^{\prime}\left(r^{\prime \prime}-1\right)\left(\frac{w t E^{\prime}}{r^{\prime}}-\frac{w t F}{r^{\prime \prime}-1}+t_{F, E^{\prime}}+1\right) \geq r^{\prime}\left(r^{\prime \prime}-1\right) \geq 0
$$

(by Lemma 2.5(2)). Thus $h^{1}\left(\operatorname{Hom}\left(F_{*}, E_{*}^{\prime}\right)\right)>0$.

Note that $\bar{h}_{i}$ is surjective and $K_{i}=\operatorname{Ker} \bar{h}_{i}$ is a proper subspace (as $\left.h^{1}\left(\operatorname{Hom}\left(F_{i *}, E_{*}^{\prime}\right)\right) \neq 0\right)$. If the extension $E_{*}$ is so chosen that the extension class

$$
[e] \notin \bigcup_{i} K_{i}
$$

(defined in Eqn. (5.9)), then $E_{*}$ cannot have a destabilizing subbundle $F_{i}$ of rank $r^{\prime \prime}-1$. This proves claim 1 .

Claim 2: $F_{*}^{\prime}=0, F_{*}=F_{*}^{\prime \prime}, h$ is a generic isomorphism.

Proof. The bundles $E_{*}^{\prime \vee}$ and $E_{*}^{\prime \prime} \vee$ are general parabolic bundles as $E_{*}^{\prime}$ and $E_{*}^{\prime \prime}$ are so. One has $s^{\text {par }}\left(E_{*}^{\vee}, E_{*}^{\prime \prime \vee}\right)=s^{\text {par }}\left(E_{*}, E_{*}^{\prime}\right)$ and $(E / F)_{*}^{\vee}$ is a subbundle of maximum slope of $E_{*}^{\vee}$. Applying the argument of Claim 1 to $E_{*}^{\vee}$ one sees that $(E / F)_{*}^{\vee} \longrightarrow E_{*}^{\prime \vee}$ is a generic surjection. Hence $E_{*}^{\prime} \longrightarrow(E / F)_{*}$ is a generic injection, i.e., $r(F) \leq r^{\prime \prime}$. From Claim $1, r(F) \geq r\left(F^{\prime \prime}\right)=r^{\prime \prime}$. Hence $r(F)=r^{\prime \prime}$, so that $F_{*} \rightarrow F_{*}^{\prime \prime}$ has zero kernel. Thus $F_{*}^{\prime}=0$, proving Claim 2 .

Since $h: F_{*} \longrightarrow E_{*}^{\prime \prime}$ is a generic isomorphism, $\mu\left(F_{*}\right) \leq \mu\left(E_{*}^{\prime \prime}\right)$. Moreover, equality implies that $h$ is an isomorphism of parabolic bundles, since $E_{*}^{\prime \prime}$ is stable and $F_{*}$ is semistable. If $h$ is an isomorphism, then the sequence splits and $E_{*}$ is not given by a generic extension. Therefore, we may assume that $\mu\left(F_{*}\right)<\mu\left(E_{*}^{\prime \prime}\right)$. By Eqn. (5.3) we have

$$
\mu\left(E_{*}^{\prime \prime}\right) \leq \mu\left(E_{*}\right)+\frac{1}{r^{\prime \prime}} \leq \mu\left(F_{*}\right)+\frac{1}{r^{\prime \prime}} .
$$

Thus $\mu\left(E_{*}^{\prime \prime}\right)-\frac{1}{r^{\prime \prime}} \leq \mu\left(F_{*}\right)<\mu\left(E_{*}^{\prime \prime}\right)$, i.e., $d\left(E_{*}^{\prime \prime}\right)-1 \leq d\left(F_{*}\right)<d\left(E_{*}^{\prime \prime}\right)$. Writing $d\left(E_{*}\right)=d(E)+w t E$ and $\delta=w^{\prime \prime}-w t F$, this gives

$$
d^{\prime \prime}-1+\delta \leq d(F)<d^{\prime \prime}+\delta .
$$

Since $h$ is a generic isomorphism, $d(F) \leq d^{\prime \prime}$. Thus $d^{\prime \prime}-1+\delta \leq d(F) \leq \operatorname{Min}\left(d^{\prime \prime}, d^{\prime \prime}+\right.$ $\delta)$. Since $w t F<r^{\prime \prime} p_{0}$, where $p_{0}$ is the number of parabolic points, one has $\delta>$ $w^{\prime \prime}-r^{\prime \prime} p_{0}$. Hence we have

$$
d^{\prime \prime}+\left[w^{\prime \prime}\right]-r^{\prime \prime} p_{0} \leq d(F) \leq d^{\prime \prime},
$$

where $\left[w^{\prime \prime}\right]$ denotes the integral part of $w^{\prime \prime}$. This shows that $d(F)$ can have only finitely many values; let $d(F)=d^{\prime \prime}-\delta^{\prime}$, where $\delta^{\prime}$ is a nonnegative bounded integer. By Eqn. (5.10), $\delta-1 \leq-\delta^{\prime}<\delta$, so

$$
-\delta^{\prime}<\delta \leq-\delta^{\prime}+1
$$


In particular,

$$
\delta+\delta^{\prime}>0
$$

Consider the exact sequence

$$
0 \longrightarrow \operatorname{Hom}\left(E_{*}^{\prime \prime}, E_{*}^{\prime}\right) \longrightarrow \operatorname{Hom}\left(F_{*}, E_{*}^{\prime}\right) \longrightarrow Q \longrightarrow 0,
$$

note that $Q$ is a torsion sheaf. The extensions of $E_{*}^{\prime \prime}$ by $E_{*}^{\prime}$ which give $E_{*}$ containing a fixed destabilizing $F_{*}$ as above are given by (parametrized by) the kernel of the homomorphism

$$
H^{1}\left(\operatorname{Hom}\left(E_{*}^{\prime \prime}, E_{*}^{\prime}\right)\right) \stackrel{\bar{h}}{\longrightarrow} H^{1}\left(\operatorname{Hom}\left(F_{*}, E_{*}^{\prime}\right)\right)
$$

induced by $h$ (defined in Eqn. (5.5)).

The cohomology exact sequence of the exact sequence Eqn. (5.12) gives

$$
H^{0}(Q) \longrightarrow H^{1}\left(\operatorname{Hom}\left(E_{*}^{\prime \prime}, E_{*}^{\prime}\right)\right) \longrightarrow H^{1}\left(\operatorname{Hom}\left(F_{*}, E_{*}^{\prime}\right)\right) \longrightarrow 0
$$

Hence $\operatorname{dim} \operatorname{Ker} \bar{h} \leq \operatorname{dim} H^{0}(Q)=\operatorname{dim} Q$, where $\bar{h}$ is defined in Eqn. (5.13).

Set

$$
a:=\sum_{p \in P} \sum_{i>j} n_{i, p}^{\prime \prime} n_{j, p}^{\prime} \text { and } b:=\sum_{p \in P} \sum_{i>j} n_{i, p}^{F} n_{j, p}^{\prime} .
$$

Since $\mu\left(E_{*}^{\prime \prime}\right)>\mu\left(E_{*}^{\prime}\right), H^{0}\left(\operatorname{Hom}\left(E_{*}^{\prime \prime}, E_{*}^{\prime}\right)\right)=0$. Hence

$$
h^{1}\left(\operatorname{Hom}\left(E_{*}^{\prime \prime}, E_{*}^{\prime}\right)\right)=-\chi\left(\operatorname{Hom}\left(E_{*}^{\prime \prime}, E_{*}^{\prime}\right)\right)=r^{\prime} d^{\prime \prime}-r^{\prime \prime} d^{\prime}+r^{\prime} r^{\prime \prime}(g-1)+a .
$$

Since $\mu\left(E_{*}\right) \leq \mu\left(F_{*}\right)$ one has

$$
r^{\prime} d^{\prime \prime}-r^{\prime \prime} d^{\prime} \geq r^{\prime \prime}\left(w^{\prime \prime}-w t F\right)+\left(r^{\prime \prime} w^{\prime}-r^{\prime} w t F\right)+r \delta^{\prime} .
$$

Hence

$$
\begin{gathered}
h^{1}\left(\operatorname{Hom}\left(E_{*}^{\prime \prime}, E_{*}^{\prime}\right)\right) \geq r^{\prime} r^{\prime \prime}(g-1)+r \delta^{\prime}+r^{\prime \prime}\left(w^{\prime \prime}-w t F\right)+\left(r^{\prime \prime} w^{\prime}-r^{\prime} w t F\right)+a \\
\geq r^{\prime} r^{\prime \prime}(g-1)+r \delta^{\prime}+r^{\prime \prime} \delta+a-b
\end{gathered}
$$

by Lemma 2.5 .

One has a commutative diagram

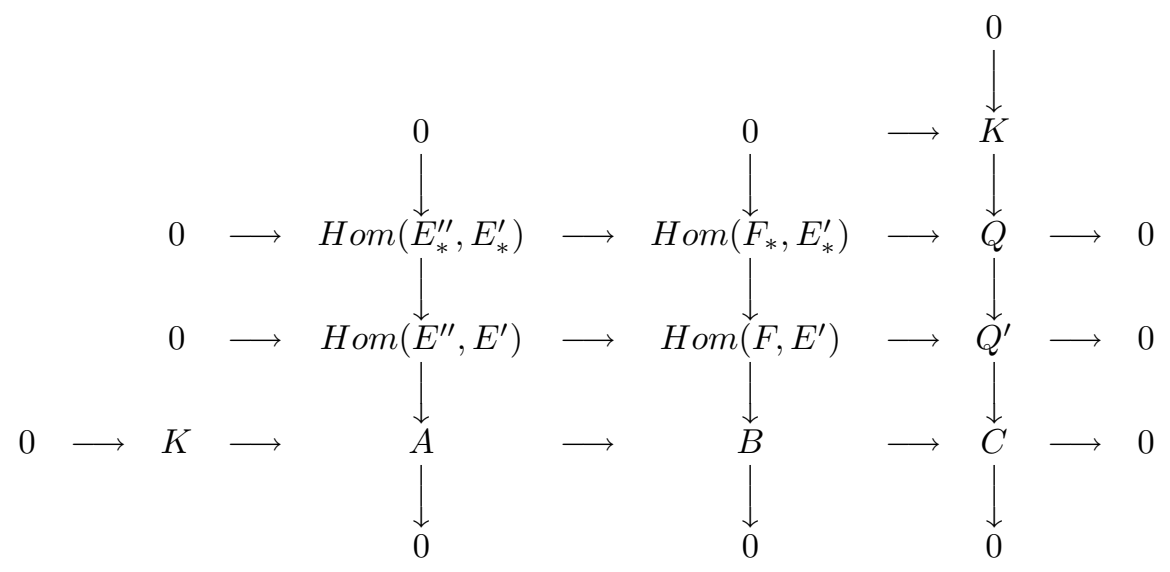


where $A, B, C, K, Q, Q^{\prime}$ are torsion sheaves, with $A, B$ and $Q^{\prime}$ of dimensions $a, b$ and $q^{\prime}=\delta^{\prime} r^{\prime}$ respectively. The bottom horizontal exact sequence gives

$$
\operatorname{dim} K-a+b-\operatorname{dim} C=0 .
$$

The last column gives

$$
\operatorname{dim} K-\operatorname{dim} Q+\delta^{\prime} r^{\prime}-\operatorname{dim} C=0 .
$$

Adding the two equations one has $a-b-\operatorname{dim} Q+\delta^{\prime} r^{\prime}=0$. i.e., $a-b=\operatorname{dim} Q-\delta^{\prime} r^{\prime}$. Using this, one has

$h^{1}\left(\operatorname{Hom}\left(E_{*}^{\prime \prime}, E_{*}^{\prime}\right)\right) \geq r^{\prime} r^{\prime \prime}(g-1)+\delta^{\prime} r+r^{\prime \prime} \delta+\operatorname{dim} Q-\delta^{\prime} r^{\prime}$

$=r^{\prime} r^{\prime \prime}(g-1)+\delta^{\prime} r^{\prime \prime}+r^{\prime \prime} \delta+\operatorname{dim} Q$

$>r^{\prime} r^{\prime \prime}(g-1)+\operatorname{dim} Q$,

as $\delta^{\prime}+\delta>0$.

Since $F_{*}$ is given by

$$
0 \longrightarrow F_{*} \longrightarrow E_{*}^{\prime \prime} \longrightarrow k^{\delta^{\prime}} \longrightarrow 0,
$$

the destabilizing subbundles $F_{*}$ (with maximum parabolic slope) are given by $\delta^{\prime} r^{\prime \prime}$ parameters. Hence extensions of $E_{*}^{\prime \prime}$ by $E_{*}^{\prime}$ which give $E_{*}$ containing such $F_{*}$ are given by parameters $N \leq \operatorname{dim} Q+\delta^{\prime} r^{\prime \prime}$. By Eqn. (5.11), one has $\delta^{\prime} \leq \delta_{0}^{\prime}=r^{\prime \prime} p_{0}-\left[w^{\prime \prime}\right]$. Hence $N \leq \operatorname{dim} Q+\delta_{0}^{\prime} r^{\prime \prime} \operatorname{Thus} h^{1}\left(\operatorname{Hom}\left(E_{*}^{\prime \prime}, E_{*}^{\prime}\right)\right)>\operatorname{dim} Q+\delta^{\prime} r^{\prime \prime}$ if $r^{\prime} r^{\prime \prime}(g-1) \geq \delta_{0}^{\prime} r^{\prime \prime}$, i.e.,

$$
g \geq 1+\frac{r^{\prime \prime} p_{0}-\left[w^{\prime \prime}\right]}{r^{\prime}} .
$$

Then a general extension of $E_{*}^{\prime \prime}$ by $E_{*}^{\prime}$ gives a stable parabolic bundle.

(B) If rank $E^{\prime \prime}=1$, then (A) holds for $g \geq 2$.

Proof. Let $\left\{\alpha_{p}^{\prime \prime}\right\}$ and $\left\{\alpha_{p}^{F}\right\}$ denote the weights of $E^{\prime \prime}$ and $F$ at $p$. Let $P^{\prime}=\{p \in$ $\left.P \mid \alpha_{p}^{F}>\alpha_{p}^{\prime \prime}\right\}$. Then

$$
\delta=\sum_{p \in P}\left(\alpha_{p}^{\prime \prime}-\alpha_{p}^{F}\right) \geq \sum_{p \in P^{\prime}}\left(\alpha_{p}^{\prime \prime}-\alpha_{p}^{F}\right) .
$$

Since $-1<\alpha_{p}^{\prime \prime}-\alpha_{p}^{F}<0$ for $p \in P^{\prime}$, one has $\delta \geq-p^{\prime}\left(p^{\prime}=\# P^{\prime}\right)$ the inequality being strict if $P^{\prime} \neq \emptyset$.

For $P^{\prime}=\emptyset$, one has wt $F_{p} \leq$ wt $E_{p}^{\prime \prime}$ for all $p \in P$, so that $\delta>0$, and $\delta^{\prime}=0$. From the proof of (A) one then has

$$
h^{1}\left(\operatorname{Hom}\left(E_{*}^{\prime \prime}, E_{*}^{\prime}\right)\right)>r^{\prime}(g-1)+\operatorname{dim} Q,
$$

$N \leq \operatorname{dim} Q$. Hence $h^{1}\left(\operatorname{Hom}\left(E_{*}^{\prime \prime}, E_{*}^{\prime}\right)\right)>N$ if $r^{\prime}(g-1) \geq 0$, i.e., $g \geq 1$.

For $P^{\prime} \neq \emptyset$, note that since $r^{\prime \prime}=1$, we have $h$ vanishes at all $p \in P^{\prime}$, and hence $\delta^{\prime} \geq p^{\prime}$. Since $\delta^{\prime} \leq 1-\delta$ and we saw that $\delta>-p^{\prime}$, one has $\delta^{\prime}<1+p^{\prime}$, i.e., $\delta^{\prime} \leq p^{\prime}$. Thus $\delta^{\prime}=p^{\prime}$ and $h$ vanishes precisely on the divisor $P^{\prime}$. Therefore, $F=E^{\prime \prime}\left(-P^{\prime}\right)$. Since $p_{0}, p^{\prime}$ are finite, $F_{*}$ varies over a finite set and hence $N \leq \operatorname{dim} Q$. It follows that $h^{1}\left(\operatorname{Hom}\left(E_{*}^{\prime \prime}, E_{*}^{\prime}\right)\right)>N$ if $g \geq 1$. This completes the proof of part (B). $\square$

Proof of Theorem 1.4. Since $M_{r^{\prime}, s} \subset V_{r^{\prime}, s}^{p a r}$, if $M_{r^{\prime}, s}$ is nonempty, then so is $V_{r^{\prime}, s}^{p a r}$. Hence part (1) follows from Proposition 5.5(2). 
Part (2) follows from Lemma 5.2(2).

By Proposition 5.6, $M_{r^{\prime}, s_{0}}$ is nonempty for $g \geq 1+\frac{r^{\prime \prime} p_{0}-\left[w^{\prime \prime}\right]}{r^{\prime}}$. Hence $V_{r^{\prime}, s_{0}}^{p a r}$ is nonempty. By Lemma 5.4(2), $V_{r^{\prime}, s}^{\text {par }}$ is nonempty for all permissible $s$. Therefore, (3) follows from Proposition 5.5(1).

\section{REFERENCES}

[BBN] Balaji, V., Biswas, I. And Nagaraj, D. S., Ramified G-bundles as parabolic bundles, Jour. Ramanujan Math. Soc., 18 (2003), pp. 123-138.

[BB] Bhosle, U. N. AND Biswas, I., Mukai-Sakai bound for equivariant principal bundles, Arkiv Math., 43 (2005), pp. 133-141.

[Bi] Biswas, I., Parabolic bundles as orbifold bundles, Duke Math. Jour., 88 (1997), pp. 305325.

[BL] Brambila-Paz, L. And Lange, H., A stratification of the moduli space of vector bundles on curves, Jour. Reine Angew. Math., 494 (1998), pp. 173-187.

[Hi] Hinschowitz, A., Problème de Brill-Noether en rang supérieur, Prepublication Mathematiques, No. 91, Nice (1986).

[KMM $]$ Kawamata, Y., Matsuda, K. and Matsuki, K., Introduction to the minimal model problem, Adv. Stud. Pure Math., 10 (1987), pp. 283-360.

[La] Lange, H., Zur Klassifikation von Regelmanigfaltigkeiten, Math. Ann., 262 (1983), pp. $447-459$.

[MS] Mukai, S. ANd Sakai, F., Maximal subbundles of a vector bundle on a curve, Manuscr. Math., 52 (1985), pp. 251-256.

[MeS] Mehta, V. B. And Seshadri, C. S., Moduli of vector bundles on curves with parabolic structures, Math. Ann., 248 (1980), pp. 205-239.

[RT] Russo, B. And Teixidor I Bigas, M., On a conjecture of Lange, Jour. Alg. Geom., 8 (1999), pp. 483-496.

[Tb] Teixidor i Bigas, M., On Lange's conjecture, Jour. Reine Angew. Math., 528 (2000), pp. $81-99$. 